\title{
Genome-wide association study identifies new locus associated with OCD
}

\begin{abstract}
Nora I. Strom ${ }^{1,2,3,4,182}$, Dongmei Yu ${ }^{5,6,182}$, Zachary F. Gerring ${ }^{7}$, Matthew W. Halvorsen ${ }^{8}$, Abdel Abdellaoui ${ }^{9}$, Cristina Rodriguez-Fontenla $^{10}$, Julia M. Sealock ${ }^{11}$, Tim Bigdeli ${ }^{12}$, Jonathan R. I. Coleman ${ }^{13,14}$, Behrang Mahjani $^{15,16}$, Jackson G. Thorp $^{17}$, Katharina Bey ${ }^{18}$, Christie L. Burton ${ }^{19}$, Jurjen J. Luykx ${ }^{20,21}$, Gwyneth Zai ${ }^{22,23}$, Kathleen D. Askland ${ }^{24}$, Cristina Barlassina $^{25}$, Judith Becker Nissen ${ }^{26,27}$, Laura Bellodi ${ }^{28}$, O. Joseph Bienvenu ${ }^{29}$, Donald Black ${ }^{30}$, Michael Bloch $^{31}$, Julia Boberg $^{32}$, Rosa Bosch ${ }^{33}$, Michael Breen ${ }^{15,34,35}$, Brian P. Brennan ${ }^{36}$, Helena Brentani ${ }^{37}$, Joseph D. Buxbaum ${ }^{15}$, Jonas
\end{abstract} Bybjerg-Grauholm ${ }^{38}$, Enda M. Byrne ${ }^{39,40}$, Beatriz Camarena ${ }^{41}$, Adrian Camarena ${ }^{42}$, Carolina Cappi ${ }^{15,37}$, Angel Carracedo ${ }^{43}$, Miguel Casas ${ }^{44,45}$, Maria C. Cavallini ${ }^{46}$, Valentina Ciullo ${ }^{47}$, Edwin H. Cook ${ }^{48}$, Vladimir Coric ${ }^{31}$, Bernadette A. Cullen ${ }^{29}$, Elles J. De Schipper ${ }^{3}$, Bernie Devlin ${ }^{49}$, Srdjan Djurovic ${ }^{50,51}$, Jason A. Elias ${ }^{36}$, Lauren Erdman ${ }^{52}$, Xavier Estivil ${ }^{53}$, Martha J.

Falkenstein $^{54}$, Bengt T. Fundin ${ }^{16}$, Maiken E. Gabrielsen ${ }^{55}$, Fernando S. Goes ${ }^{29}$, Marco A. Grados ${ }^{29}$, Jakob Grove ${ }^{56,57}$, Wei Guo $^{58,59}$, Jan Haavik ${ }^{60,61}$, Kristen Hagen ${ }^{62,63}$, Alexandra Havdahl ${ }^{64,65,66}$, Ana G. Hounie ${ }^{37}$, Donald Hucks ${ }^{11,67}$, Christina Hultman $^{16}$, Magdalena Janecka ${ }^{15,34}$, Michael Jenike ${ }^{68}$, Elinor K. Karlsson ${ }^{69,70}$, Julia Klawohn ${ }^{1}$, Lambertus Klei ${ }^{71}$, Janice Krasnow $^{72}$, Kristi Krebs ${ }^{73}$, Jason Krompinger ${ }^{36}$, Nuria Lanzagorta ${ }^{74}$, Fabio Macciardi ${ }^{75}$, Brion Maher ${ }^{76}$, Evonne McArthur ${ }^{11}$, Nathaniel McGregor ${ }^{77}$, Nicole C. McLaughlin ${ }^{78}$, Sandra Meier ${ }^{79}$, Euripedes C. Miguel ${ }^{37}$, Maureen Mulhern ${ }^{15,34}$, Paul S. Nestadt $^{29}$, Erika L. Nurmi ${ }^{80}$, Kevin S. O'Connell ${ }^{81,82}$, Lisa Osiecki ${ }^{5,83}$, Teemu Palviainen ${ }^{84}$, Fabrizio Piras ${ }^{47}$, Federica Piras ${ }^{47}$, Ann E. Pulver ${ }^{29}$, Raquel Rabionet ${ }^{53}$, Alfredo Ramirez ${ }^{85,86,87,88}$, Scott Rauch $^{54}$, Abraham Reichenberg ${ }^{89}$, Jennifer Reichert ${ }^{15,34}$, Mark A. Riddle ${ }^{29}$, Stephan Ripke ${ }^{6,90,91}$, Aline S. Sampaio ${ }^{37,92}$, Miriam A. Schiele $^{93}$, Laura G. Sloofman ${ }^{15}$, Jan Smit ${ }^{94}$, Janet L. Sobell $^{95}$, María Soler Artigas ${ }^{96,97,98,99}$, Laurent F. Thomas ${ }^{100,101}$, Homero Vallada ${ }^{37,102}$, Jeremy Veenstra-VanderWeele ${ }^{103}$,

Nienke N. C. C. Vulink ${ }^{9}$, Christopher P. Walker ${ }^{104}$, Ying Wang ${ }^{29}$, Jens R. Wendland ${ }^{105}$, Bendik S. Winsvold ${ }^{106,107,108}$, Yin

Yao $^{109}$, Pino Alonso ${ }^{110}$, Götz Berberich ${ }^{111}$, Cynthia M. Bulik ${ }^{16,112,113}$, Danielle Cath ${ }^{114,115}$, Daniele Cusi ${ }^{116}$, Richard

Delorme $^{117}$, Damiaan Denys ${ }^{118}$, Valsamma Eapen ${ }^{119}$, Peter Falkai ${ }^{120}$, Thomas V. Fernandez $^{31}$, Abby J. Fyer ${ }^{121,122}$, Daniel A. Geller $^{5,123}$, Hans J. Grabe ${ }^{124}$, Benjamin D. Greenberg ${ }^{77,78,125}$, Gregory L. Hanna ${ }^{126}$, lan M. Hickie ${ }^{127}$, David M. Hougaard ${ }^{38,57}$, Norbert Kathmann ${ }^{1}$, James Kennedy ${ }^{23}$, Liang Kung-Yee ${ }^{128,129}$, Mikael Landén ${ }^{16,130}$, Stéphanie Le Hellard ${ }^{131,132}$, Marion Leboyer $^{133}$, Christine Lochner ${ }^{134}$, James T. McCracken ${ }^{80}$, Sarah E. Medland ${ }^{7}$, Preben B. Mortensen ${ }^{57,135,136}$, Benjamin Neale $^{83,137,138}$, Humberto Nicolini ${ }^{139,140}$, Merete Nordentoft ${ }^{141,142}$, Michele Pato ${ }^{143}$, Carlos Pato ${ }^{143}$, David L. Pauls ${ }^{144}$, Nancy L. Pedersen $^{16}$, John Piacentini ${ }^{80}$, Christopher Pittenger ${ }^{145}$, Danielle Posthuma ${ }^{146}$, Josep A Ramos-Quiroga ${ }^{147,148,149,150}$, Steven A. Rasmussen ${ }^{78}$, Kerry J. Ressler ${ }^{36}$, Margaret A. Richter ${ }^{23,151}$, Maria C. Rosário ${ }^{152}$, David R. Rosenberg ${ }^{153}$, Stephan Ruhrmann $^{85}$, Jack F. Samuels ${ }^{29}$, Sven Sandin ${ }^{15,16}$, Paul Sandor ${ }^{23}$, Gianfranco Spalletta ${ }^{47,154}{ }^{\text {, Dan J. Stein }}{ }^{155}$, S. Evelyn

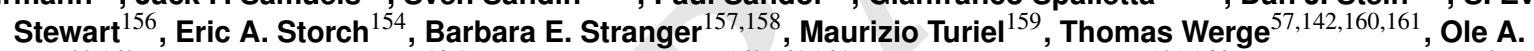

Andreassen $^{82,162}$, Anders D. Børglum ${ }^{56,57}$, Susanne Walitza ${ }^{163,164,165}$, Bjarne K. A. Hansen ${ }^{131,166}$, Christian P. Rück ${ }^{3}$, Nicholas G. Martin ${ }^{17}$, Lili Milani ${ }^{73}$, Ole Mors ${ }^{167}$, Ted Reichborn-Kjennerud ${ }^{64,162}$, Marta Ribasés ${ }^{97,168,169,170}$, Gerd Kvale ${ }^{131,166}$, David Mataix-Cols $^{3}$, Katharina Domschke ${ }^{93,171}$, Edna Grünblatt ${ }^{163,164,165}$, Michael Wagner ${ }^{18}$, John-Anker Zwart ${ }^{106,107,172}$, Gerome

Breen $^{13,14}$, Gerald Nestadt ${ }^{29}$, Andres Metspalu ${ }^{73}$, Jaakko Kaprio ${ }^{173}$, Paul D. Arnold ${ }^{174,175}$, Dorothy E. Grice ${ }^{15}$, James A. Knowles $^{176}$, Helga Ask ${ }^{64}$, Karin J. H. Verweij ${ }^{9}$, Lea K. Davis ${ }^{67}$, Dirk J. A. Smit ${ }^{177}$, James J. Crowley ${ }^{3,8,112}$, Carol A. Mathews ${ }^{178}$, Eske M. Derks $^{17}$, Jeremiah M. Scharf ${ }^{5,6,183}$, and Manuel Mattheisen ${ }^{4,180,181,183}$

${ }^{1-181}$ Affiliations appear at the end of the document

${ }^{182,183}$ Authors contributed equally

Obsessive-compulsive disorder (OCD) is a heritable disorder, but no definitive, replicated OCD susceptibility loci have yet been identified by any genome-wide association study (GWAS). Here, we report results from a GWAS in the largest OCD casecontrol sample $(N=14,140$ OCD cases and $N=562,117$ controls) to date. We explored the genetic architecture of $\mathrm{OCD}$, including its genetic relationships to other psychiatric and nonpsychiatric phenotypes. In the GWAS analysis, we identified one SNP associated with $O C D$ at a genome-wide significant level. Subsequent gene-based analyses identified additional two genes as potentially implicated in OCD pathogenesis. All SNPs combined explained $16 \%$ of the heritability of OCD. We show substantial positive genetic correlations between $O C D$ and a range of psychiatric disorders, including anxiety disorders, anorexia nervosa, and major depression. We thus for the first time provide evidence of a genome-wide locus implicated in OCD and strengthen previous literature suggesting a polygenic nature of this disorder.

obsessive-compulsive disorder | OCD | GWAS

Correspondence: Manuel Mattheisen, Department of Psychiatry, Dalhousie University, Halifax, Canada, manuel.mattheisen@dal.ca

\section{Introduction}

Obsessive-compulsive disorder (OCD) is a chronic mental illness that affects approximately $2-3 \%$ of the general population $^{1 ; 2}$. It is characterized by obsessions and compulsions that vary in type and severity across patients, with most showing a waxing and waning illness course. Age at onset is usually in early to late adolescence but diagnosis and initiation of treatment are often delayed by several years $3 ; 4$. OCD frequently co-occurs with other disorders, particularly tic disorders, eating disorders, depressive disorders, and anx-

NOTE: This preprint reports new research that has not been certified by peer review and should not be used to guide clinical practice. 
iety disorders ${ }^{5-8}$. Although anxiety is a common symptom of OCD, OCD is currently considered to be distinct from anxiety disorders in terms of its epidemiological, clinical and pathophysiological presentation. This uniqueness is recognized in DSM-5 and ICD11, which separate OCD, along with several related disorders, into a separate disorder category, Obsessive Compulsive and Related Disorders (OCRDs) ${ }^{2}$.

Although its pathogenesis is yet to be fully elucidated, it is now clear that complex genetic factors play a role in the susceptibility to and/or development of OCD. The heritability of obsessive-compulsive (OC) symptoms as estimated by twin studies is between 27 and $47 \%$ in adults and between 45 and $65 \%$ in children ${ }^{9-11}$. SNP-based heritability of OCD, estimated using genome complex trait analysis (GCTA) is between $28-37 \%$, with higher estimates for childhood-onset OCD, in line with heritability estimates from twin studies ${ }^{12 ; 13}$. GCTA analyses further indicate that much of the genetic risk for OCD arises from common variation in multiple loci, each with a small effect, acting in concert $^{12}$. Although rare variants exerting larger effects may also play a role in OCD development ${ }^{14-17}$, less work has been done in this area.

Two genome-wide association studies (GWAS) of OCD as well as a combined meta-analysis of 2,688 cases and 7,037 controls have been published to date ${ }^{13 ; 18 ; 19}$. However, all three studies were significantly under-powered based on estimates that suggest a need for tens to hundreds of thousands of individuals (cases and controls) for definitive gene identification in psychiatric disorders with complex inheritance ${ }^{20}$. Accordingly, no definitive, replicated OCD susceptibility loci have yet been identified by any study. Nevertheless, while small, these prior GWASs did demonstrate significant SNP-based heritability, indicating that a well-powered OCD GWAS with a much larger sample size would likely identify genome-wide significant loci and more accurate SNP effects, similar to what has been demonstrated for other major psychiatric disorders ${ }^{21-23}$. Such findings will provide insight into the biological underpinnings of OCD and may improve follow-up analyses and contribute to (multimodal) models for risk prediction. In addition, the frequent co-occurrence seen between OCD and other psychiatric illnesses appears to be due, at least in part, to shared genetic susceptibility. Crossdisorder studies demonstrate that OCD is genetically correlated with a range of other psychiatric disorders, most notably Tourette syndrome (TS), anorexia nervosa (AN), and major depressive disorder (MDD) $)^{24 ; 25}$, all of which are highly comorbid with OCD.

Here, we performed a GWAS in a substantially expanded case-control sample $(\mathrm{N}=14,140 \mathrm{OCD}$ cases and $\mathrm{N}=562,117$ controls). We explored the genetic architecture of OCD, including its genetic relationships to other psychiatric and nonpsychiatric phenotypes, such as TS, AN, and MDD. In the GWAS analysis, we identified one SNP associated with OCD at a genome-wide significant level. Subsequent gene-based analyses nominated two additional genes as potentially implicated in OCD pathogenesis. All SNPs combined explained $16 \%$ of the heritability of OCD. We also show substantial positive genetic correlations between OCD and a range of psychiatric disorders, including those with anxiety disorders and MDD.

\section{Methods}

\section{Samples}

We analyzed genomic data from 17 OCD case-control cohorts (11,312 OCD cases and 557,230 controls in total) not included in any previous OCD GWAS publications. In addition, three previously published GWAS datasets (two from the International OCD Foundation-Genetics Consortium (IOCDF-GC) ${ }^{18}$ and one from The OCD Collaborative Geneticws Association Study (OCGAS) ${ }^{19}$ ) were re-analyzed using newly matched control samples that were genotyped with the same microarrays as the cases $(2,828$ cases and 4,887 controls). In total, 20 cohorts comprising 14,140 OCD cases and 562,117 controls of European ancestry were included in the analyses. Among these, 323 cases were part of a parentproband trio; in these cases, parents were used as pseudo controls. A total of 12,607 cases met DSM-IV ${ }^{26}$ or ICD10 criteria for OCD, while the remaining 1,533 cases were based on self-reported OCD diagnosis. Supplementary Table S1 provides an overview of the individual cohorts, cohort-specific sample and analytic details can be found in the Supplementary data. Data collections were approved by the relevant institutional review boards at all participating sites, and all participants provided written informed consent.

\section{Genetic data formatting, cleaning, alignment, and individual GWASs}

First, the data of each participating cohort were analyzed individually (see Supplementary Methods for details). Genetic data were imputed using either the Haplotype Reference Consortium (HRC) ${ }^{27}$ or 1000 Genomes Project Phase 3 reference panels ${ }^{28}$. The resulting GWAS summary statistics were then harmonized before a conjoint meta-analysis was conducted. Each summary statistic data set was transformed to 'daner' file format following ricopili ${ }^{29}$ specifications. Next, each dataset was cleaned of variants that were likely to have poor underlying genotype data. All variants had to meet the following criteria for inclusion: minor allele frequency $(\mathrm{MAF})>1 \%$ in cases and controls, imputationquality (INFO) score $>0.8$ and $<1.2$. If the effect measure, $p$-value or standard error (SE) was missing or was out of bounds (infinite), the SNP was removed. Only biallelic SNPs were retained in the data. Once cleaned summary statistics were produced, all datasets were aligned to the HRC reference panel ${ }^{27}$. If variants were reported on different strands, they were flipped to the orientation in the HRC-reference. Furthermore, strand-ambiguous $\mathrm{A} / \mathrm{T}$ and $\mathrm{C} / \mathrm{G}$ SNPs were removed if their MAF was $>0.4$. In case A/T and C/G SNPs showed a MAF $<0.4$ allele frequencies were compared to frequencies in the HRC-reference. If an allele frequency 
match was found, i.e., the respective allele was also the minor allele in the $\mathrm{HRC}$ reference, same strand orientation was assumed. If an allele mismatch was found, i.e. the allele had a frequency $>0.5$ in HRC, it was assumed that alleles were reported on different strands and alleles were flipped subsequently. Marker-names were uniformly switched to those present in the $\mathrm{HRC}$ reference. If a variant did not overlap with the variants in the HRC reference, it was removed.

\section{Genome-wide association meta-analysis}

Inverse variance weighted meta-analysis was conducted on 20 European cohorts using METAL ${ }^{30}$. Heterogeneity was assessed with Cochran's $I^{2}$ statistic. The genomic control factor $\left(\lambda_{1000}\right)$ was calculated for each individual GWAS and for the overall meta-analysis to identify residual population stratification or systematic technical artifact. GWAS summary statistics were subjected to linkage disequilibrium (LD) score regression (LDSC) analyses on high-quality common SNPs (INFO score > 0.9) to examine the LDSC intercept as an alternative evaluation of test statistic inflation due to residual artifact or population stratification, and to estimate the genetic heritability from the meta-analysis and genetic correlations between cohorts. The genome-wide significance threshold for the GWAS was set at a p-value of $5.0 \times 10^{-8}$. Sign tests on the top SNPs (inclusion threshold of $p=0.0001, p=0.00001, p=0.000001$, and $p=5.00 \times 10^{-8}$ ) were performed between each individual cohort as well as 'leave-one-out' meta-analyses to identify any cohort in which the summary statistics significantly deviated from the rest of the cohorts.

\section{Polygenic risk scoring}

OCD polygenic risk scores (PRS) were calculated to assess the predictive value of the new meta-analysis. For this purpose, we conducted three 'leave-one-out' GWAS metaanalyses that each excluded one of three samples (IOCDFGC, OCGAS, and Psych_Broad) and tested how well the PRS predicted case-control status in the excluded sample. We selected these three datasets based on their previous involvement in a recent meta-analysis (IOCDF-GC and OCGAS; see above) and their availability at the site that conducted the analysis (Psych_Broad). PRS were generated at eight $\mathrm{p}$-value thresholds $(0.001,0.05,0.1,0.2,0.3,0.4,0.5$, and 1) as a weighted sum of the risk allele dosages. The contribution of PRS was measured by comparing Nagelkerke's preudo- $R^{2}$ of the full model (including principal components (PCs), sex, and PRS) and of the reduced model (adjusted for PCs and sex).

\section{Gene-based analyses}

Conventional MAGMA (C-MAGMA) We used conventional MAGMA (C-MAGMA v1.07) ${ }^{31}$ to test genetic associations at the gene level for the combined effect of SNPs in or near protein-coding genes while adjusting for LD between the SNPs and the size of the gene. We used the 1000 Genomes reference panel (Phase 3$)^{28}$ to control for LD. SNPs were mapped to genes if they were located within $10 \mathrm{~kb}$ of the gene coding region. The significance threshold was set using Bonferroni correction (i.e. 0.05/20,031 tests performed: $p<$ $\left.2.50 \times 10^{-6}\right)$.

Expression informed analysis (E-MAGMA, S-PrediXcan, S-MultiXcan) We conducted gene-based tests informed by expression quantitative trait loci (eQTL) to identify genes that are enriched for association signal. We used a novel eQTL-informed gene-based test (E-MAGMA) ${ }^{32}$ that assigns SNPs to genes based on significant associations (FDRcorrected $p<0.05$ ) with tissue-specific eQTLs in 48 GTEx tissues (v8). E-MAGMA is conducted in MAGMA (v1.07) by modifying gene annotation files by integration of eQTL information and uses the 1000 Genomes reference panel (Phase 3) to model LD. The significance threshold was set using Bonferroni correction across all tissues at $p<$ $2.51 \times 10^{-7}$ (i.e. $0.05 / 199,421$ tests performed).

We used S-PrediXcan ${ }^{33}$ to integrate expression quantitative trait loci (eQTL) information with our GWAS summary statistics and identify genes whose genetically predicted expression levels were associated with OCD. S-PrediXcan estimates gene expression weights by training a linear prediction model in samples with both gene expression and SNP genotype data. The weights are then used to predict gene expression from GWAS summary statistics, while incorporating the variance and covariance of SNPs from an LD reference panel. We used expression weights for 48 tissues from the GTEx Project (v8) ${ }^{34}$ and LD information from the 1000 Genomes Project (Phase 3$)^{28}$ (see web resources). These data were integrated with $\beta$ values and standard errors from the OCD GWAS meta-analysis to test the association between imputed levels of gene expression and OCD risk. The significance threshold was set at $p<1.67 \times 10^{-7}$ using Bonferroni correction (i.e. 0.05/299,996). Next, we used S-MultiXcan, which integrates the Predixcan associations across the 48 GTEx tissues into a single test, thus maximizing statistical power. We applied a transcriptome-wide significance threshold of $p<$ $2.31 \times 10^{-6}$ (i.e. $0.05 / 21,601$ tests).

Chromatin informed (H-MAGMA) We further conducted gene-based tests that integrated genetic results with $\mathrm{Hi}-\mathrm{C}$ information using H-MAGMA ${ }^{35}$. SNPs were assigned to genes by leveraging regulatory (chromatin interaction) relationships in human brain tissue. H-MAGMA is conducted in MAGMA (v1.07) using the 1000 Genomes reference panel (Phase 3) to model LD. The significance threshold was set using Bonferroni correction (i.e. 0.05/24,358: $p<2.05 \times 10^{-6}$ ).

Functional annotation of GWAS findings We also performed an extensive functional annotation for all SNPs in genomic areas identified by our lead SNPs using FUMA (see web-resources) ${ }^{36}$ to identify the most likely causal variants. In addition, the list of genes significantly associated with OCD, as indicated by at least one of the four gene-based methods (Table 2; except LOC101928274 as it is neither contained in the gene catalog of FUMA nor in the ex- 
pression data) was used to create expression heatmaps to visualize the average expression value of the associated genes. Further, a differential gene expression analysis (DEG) was performed to test the expression of associated genes against all other genes for each expression data set (tissue and developmental stage) using GTEx v8 and BrainSpan data ${ }^{37}$.

\section{Tissue and cell-type enrichment analysis}

To determine if there are specific tissues or cell-types whose gene expression profiles are enriched for OCD risk variation, we used an analysis protocol recently described in Bryois et al. ${ }^{38}$. Consistent with this we utilized their code-base for this analysis, and in particular the sets of genes that mark different highlighted tissue and cell-type datasets (see web-resources for data source).

We selected 3 datasets that had been preprocessed by Bryois et al. ${ }^{38}$ for inclusion in this analysis. The first features tissue-specific gene expression data derived from GTEx ${ }^{39}$, with a total of 37 tissues represented. The second and third datasets are derived from Zeisel et al. ${ }^{40}$, and represent 1) broad cell-type groups across the entirety of the mouse nervous system, and 2) a high-resolution single cell-type map of the same data. In Zeisel et al. ${ }^{40}$, a total of 39 broad cell-type groups and 265 individual cell-types are represented.

We followed the analysis protocol described in Bryois et al. ${ }^{38}$ for the analyses of 37 tissues from GTEx and 39 broad celltype groups from Zeisel et al. ${ }^{40}$, and utilized a simplified approach for the analysis of each of the 265 individual celltypes from Zeisel et al. ${ }^{40}$. For the tissue and broad cell-type group datasets, we conducted the full protocol from Bryois et $\mathrm{al}^{38}$ which included analyzing tissue/cell-type enrichment using both $\operatorname{LDSC}^{41}$ and MAGMA ${ }^{31}$. For each dataset, pvalues were adjusted for a false discovery rate of $5 \%$. We only considered a tissue or cell-type significantly enriched if the FDR-adjusted p-value was less than 0.05 in both the LDSC and MAGMA-based tests. Due to the high computational demands of analysing 265 individual cell-types across the mouse nervous system in LDSC, we limited the assessment protocol to using MAGMA only, and considered a celltype as significant if it had an FDR-adjusted p-value of less than 0.05. All statistical analyses downstream of LDSC and MAGMA (namely, p-value adjustment) were done using R v3.6.1, and all plotting was done inside of $\mathrm{R} v 3.6 .1$ using the package ggplot2 $23.2 .1^{42}$.

\section{Overlap of the genome-wide significant locus with high-confidence chromatin interactions}

To derive chromatin interactions found in brain tissue that overlap with the genome-wide significant region(s) from the OCD GWAS, we submitted a corresponding analysis job to FUMA $^{36}$ on May 3rd 2020 using the OCD GWAS sumstats as input. We selected the following chromatin interaction datasets for inclusion: 1) Promoter anchored loops from the PsychENCODE project ${ }^{43}, 2$ ) loops from fetal cortex tissue, from Giusti-Rodriguez et al. $2019^{44}$, and 3) loops from adult cortex tissue, also from Giusti-Rodriguez et al.
$2019^{44}$. All other parameters were set to default settings (SNP2GENE: a) maximum p-value of lead SNPs $<5 \times 10^{-8}$, maximum $\mathrm{p}$-value cutoff $<.05, r^{2}$ threshold to define independent significant SNPs $\geq .6$, second $r^{2}$ threshold to define lead SNPs $\geq .1$, reference population: $1000 \mathrm{G}$ phase 3 EUR, maximum distance between LD blocks to merge into a locus $<250 \mathrm{~kb}$. b) Positional gene mapping: distance to genes or functional consequences of SNPs on genes to map: $50 \mathrm{~kb}$, SNP filtering: minimum CADD score $\geq 12.37$, maximum RegulomeDB score $=7$, annotation datasets eQTL catalogue: PsychENCODE, FANTOM5, Brain Open Chromatin Atlas. c) Gene eQTL mapping: Tissue types from BrainSeq Brain, BRAINEAC, GTEx v8 Brain, eQTL; eQTL pvalue threshold: only significant snp-gene pairs with FDR $<.05$. GENE2FUNC: all background genes used, gene expression datasets: GTEx v8:54 tissue types, 30 general tissue types, 29 different ages of brain samples, and 11 general developmental stages of brain samples, Benjamin-Hochberg (FDR) correction was used to test for multiple testing in geneenrichment analyses, maximum adjusted p-value for gene set association $<.05$, minimum overlapping genes with gene sets $\geq 2$ ).

\section{SNP-based heritability and genetic correla- tion with other traits}

The proportion of variance in liability to OCD that could be explained by the aggregated effect of all included SNPs (SNP-based heritability) was estimated using LDSC $^{41}$. The SNP heritability is based on the estimated slope from the regression of the SNP effect sizes from the GWAS on the LD score. Genetic correlations between OCD and 82 other disorders/traits of potential relevance to OCD were estimated in cross-trait LDSC, a method that computes genetic correlations between GWASs without bias from ancestry differences or sample overlap ${ }^{45}$. The genetic correlation between traits is based on the estimated slope from the regression of the product of Z-scores from two GWASs on the LD score and represents the genetic covariation between two traits based on all polygenic effects captured by the included SNPs. The source studies of the GWAS summary statistics used are summarized in Supplementary Table S9. The genome-wide LD information used by these methods was based on European populations from the HapMap 3 reference panel ${ }^{45 ; 46}$ (see web resources). The GWAS summary statistics were filtered to only include SNPs that were part of the 1,290,028 million genome-wide HapMap 3 SNPs used in the original LD score regression studies ${ }^{45 ; 46}$. As a follow-up, we repeated the same analyses separately for three OCD subsets: 1) including only clinical samples, 2) including only (large-scale) biobank samples, and 3) including only data sets that were not primarily ascertained for OCD (AUS and iPSYCH).

\section{Results}

\section{Genome-wide association results}

Prior to conducting the GWAS meta-analysis, QQ plots and genomic inflation factors (reported in Supplementary Table 
S1 as $\lambda 1000)$ from each of the individual GWAS data sets were evaluated, and no evidence for significant residual population stratification was observed. The final GWAS meta-analysis contained 20 data sets consisting of 14,140 OCD cases, 562,117 controls and 7,027,156 autosomal SNPs. No significant residual stratification effects were observed (Supplementary Figure S1 QQ plot; $\lambda=1.151$; $\left.\lambda_{1000}=1.005\right)$. One SNP ( $\mathrm{rs} 2581789, p=2.1 \times 10^{-8}, \mathrm{OR}=$ $0.92)$ exceeded the genome-wide threshold for significance (See Figure 1 for Manhattan plot, Figure 2 for regional plot of rs2581789, and Figure 3 for a forest plot showing the effect sizes in each individual cohort for rs2581789). This SNP is located in the intergenic region between SFMBTI and RFT1 on chromosome 3p21.1. The LD block tagged by rs2581789 spans $325.8 \mathrm{~kb}\left(\mathrm{LD} \mathrm{r}{ }^{2}>0.6\right)$ and encompasses 12 genes, including NEK4, ITIH1, ITIH3, ITIH4, ITIH4-AS1, MUSTN1, STIMATE-MUSTN1, STIMATE, MIR8064, SFMBT1, RFT1, and PRKCD. Eight additional independent GWAS loci with p-values $<1.0 \times 10^{-5}$ were identified (corresponding association data, genomic regions, and genes in LD with the lead SNP are reported in Table 1, see Supplementary Figure S2-S9 for regional plots and Supplementary Figures S10-S17 for forest plots of each SNP).

This SNP (rs2581789) has previously been reported to be associated with schizophrenia (SCZ) ${ }^{47}$ and a combined SCZ/bipolar disorder (BP) phenotype ${ }^{48}$, as well as with anthropomorphic traits such as body mass index (BMI) ${ }^{49}$ and waist-hip-ratio ${ }^{49}$, and with psychological traits such as worry ${ }^{50-52}$ and well-being ${ }^{53}$. Other top-ranked SNPs identified in this analysis have also been previously reported to be significantly associated with psychological traits such as anxiety ${ }^{54}$, depressive symptoms ${ }^{53}$, neuroticism ${ }^{50 ; 52 ; 53 ; 55}$, hurt ${ }^{50}$, worry too long after embarrassment ${ }^{50 ; 52}$, nervous feelings ${ }^{50}$ and worry/vulnerability ${ }^{54}$, as well as with cognitive performance and educational attainment ${ }^{56}$ (see Supplementary Table S2 for a full list of previously reported significant associations of the top nine SNPs identified in the OCD GWAS).

\section{Polygenic risk scoring}

In the 'leave-one-out' PRS analyses we observed significantly higher OCD PRS among OCD cases compared to the controls in each target sample, explaining 3.9\%, 3.5\%, and $3.3 \%$ of overall phenotypic variance of IOCDF-GC, OCGAS, and Psych_Broad, respectively (Supplementary Figure S18).

\section{Gene-based analyses}

We identified 18 genes that were significantly associated with OCD in at least one of the four gene-based methods (C-MAGMA, E-MAGMA, H-MAGMA, and S-PrediXcan) following correction for multiple hypothesis testing at the experiment-wide or transcriptome-wide level (see Table 2 for a list of all significant genes; full results can be found in Supplementary Tables S3, S4, S5, and S6). Genes identified by the four methods partially overlapped (Venn-diagram,
Supplementary Figure S19). C-MAGMA revealed five genes significantly associated with OCD : PER1, SFMBTI, CTC1, TMEM107, and NEFH at a Bonferroni-corrected significance threshold of $p<2.50 \times 10^{-6}$ (Supplementary Table S3). HMAGMA identified 14 risk genes across 7 genomic regions associated with OCD (Bonferroni-corrected $p<2.05 \times 10^{-6}$; Supplementary Table S4). Using E-MAGMA, we identified one gene that was significantly associated with OCD (CTC1 in whole blood, $p<2.51 \times 10^{-7}$, Supplementary Table S5). CTC1 was also the most significantly associated gene using S-PrediXcan (whole blood, $p=3.04 \times 10^{-7}$ ), but did not survive multiple testing correction in this analysis $\left(p<1.78 \times 10^{-7}\right)$ (Supplementary Table S6). We therefore meta-analyzed the tissue-specific S-PrediXcan associations (i.e. combining association signals across 48 GTEx tissues) using S-MultiXcan to generate a single test statistic (see Supplementary Table S7) for each gene. This approach yielded two significant $\left(p<2.31 \times 10^{-6}\right)$ genes: TMEM107 $(p=$ $\left.2.44 \times 10^{-7}\right)$ and $R P L 35\left(p=1.01 \times 10^{-6}\right)$.

\section{Functional annotation of GWAS findings}

The functional annotation of individual GWAS findings (FUMA) is presented in Supplementary Table S8) and in the regional annotation plot for the genome-wide significant chromosome 3 locus (see Supplementary Figure S20). We explored tissue-specific expression patterns for 17 of the 18 genes resulting from the gene-based tests (excluding LOC101928274 for which no information was available). Results are visualized for the 54 GTEx tissues (see Supplementary Figure S21) and for Brainspan gene expression data (Supplementary Figures S22 and S23). Deferentially Expressed Gene (DEG) set analyses were conducted to test whether the set of 17 genes implicated by one or more genebased tests are significantly up-regulated or down-regulated in any of the tissues. No significant differences were found (Supplementary Figures S24, S25, and S26).

\section{Tissue and cell-type enrichment analyses}

We performed association tests for enrichment between OCD GWAS summary statistics and three gene expression profiles from human tissues and mouse cell-types. No tissue or cell-type was significantly associated with OCD after FDR correction for both the LDSC and MAGMA methods (Supplementary Figure S27A), thus failing to reach our criterion for significance. Nevertheless, tissues derived from brain regions clustered preferentially at the top of the results distribution. Also, no broad cell-type category reached significance for both LDSC and MAGMA (Supplementary Figure S27B). Here, the top-ranking result was 'telencephalon projecting excitatory neurons'. A further eight types included three types of di- and mesencephalon inhibitory neurons from the midbrain, two types of di- and mesencephalon excitatory neurons (one from thalamus and one from midbrain), and two types of telencephalon inhibitory interneurons from the hippocampus/cortex. In the high-resolution single celltype analysis using MAGMA, nine specific cell-types were significantly enriched for OCD after FDR-correction (Sup- 
plementary Figure S28).

\section{Overlap of the genome-wide significant locus with high-confidence chromatin interactions}

Genome-wide significant hits in other psychiatric GWAS studies have been shown to be enriched in overlap with brain-specific chromatin interactions ${ }^{44 ; 57}$, and we sought to determine if this overlap (and potential subsequent mechanism of effect) existed for the genome-wide significant locus. We utilized FUMA ${ }^{36}$ to determine if the genome-wide significant locus from the GWAS (lead SNP located at hg 19 chr3:53096230) overlapped with any high-confidence chromatin interactions derived from either PsychENCODE ${ }^{57}$ or fetal and adult cortex from Giusti-Rodriguez et al. ${ }^{44}$. While several loops connected the locus with a distal gene on the same chromosome (see Supplementary Figure S29), followup analyses did not reveal any genes with an obvious dosage sensitivity.

\section{SNP-based heritability and genetic correla- tions with other traits}

The SNP-based heritability of OCD as obtained from LDscore regression was estimated to be 0.164 ( $\mathrm{SE}=0.012$ ). In genetic correlation analyses between OCD and summary statistics from 82 behavioral, cognitive, psychiatric, neurological, allergic/immunologic, metabolic, and anthropomorphic traits 31 of the 82 traits investigated had significant genetic correlations after correction for multiple testing (Figure 4 and Supplementary Table S9). In particular, OCD showed significant positive genetic correlations with all psychiatric disorders, with especially high correlations with anxiety disorder $\left(r_{g}=0.627, S E=0.057\right.$, FDR-corrected $p=$ $6.11 \times 10^{-27}$, AN $\left(r_{g}=0.588, S E=0.04\right.$, FDR-corrected $p$ $\left.=1.91 \times 10^{-48}\right)$, and MDD $\left(r_{g}=0.54, S E=0.042\right.$, FDRcorrected $\left.p=6.58 \times 10^{-36}\right)$. OCD was also positively correlated with alcohol dependence, while cannabis use disorder and other substance-use traits did not show significant correlations. Moreover, OCD was significantly genetically correlated with several cognitive/SES related traits, including a positive correlation with memory and negative correlations with intelligence, income, and job satisfaction. Also, some of the anthropomorphic traits (BMI, waist-hip-circumference, and hip-circumference) and auto-immune disorders (Crohn's disease, ulcerative colitis, and inflammatory bowel disease) showed a significant negative correlation with OCD. In addition, significant correlations of substantial effect $(r>|0.25|)$ were found with neuroticism, suicidality, tiredness (all positive), and with subjective well-being (negative). Somewhat less prominent, but also significant was the positive correlation with childhood maltreatment. Genetic correlations with neurological, substance use, cardiovascular, and fertility traits were weak and non-significant.

As follow-up sensitivity analyses, we repeated the genetic correlation analyses for clinically-ascertained samples and biobank samples separately, as well as for the full OCD GWAS sample excluding the two datasets that were not primarily ascertained for OCD (AUS and DOTS). The resulting correlations were similar, though not identical, across these three OCD sub-samples (see Supplementary Figure S30). As an example for differences in the sub-samples, only the clinically-ascertained OCD samples had significant genetic correlations with ADHD and PTSD. Generally, the biobankderived OCD samples showed less pronounced correlations than the other two subsets, which might be expected due to their smaller case sample sizes.

\section{Discussion}

In this GWAS, containing approximately five times the number of individuals diagnosed with OCD than previous studies ${ }^{13 ; 18 ; 19}$, we report the first definitive genome-wide significant locus for OCD (rs2581789 on chromosome 3p21.1). All SNPs combined in our analysis explained $16 \%$ of the heritability of OCD. We also showed substantial positive genetic correlations between OCD and a range of psychiatric disorders, including anxiety disorders, AN, and MDD.

Our top SNP rs2581789 resides in a gene-rich genomic region (see Figure 2 for a regional association plot and a list of genes in the region). This region has previously been associated with a broad range of other psychiatric disorders and related traits, including $\mathrm{SCZ}^{47}$, well-being ${ }^{52}$, and the worry-subcluster of neuroticism ${ }^{50}$. Of note, this SNP was also identified in a recent cross-disorder meta-analysis of eight psychiatric disorders from the Psychiatric Genomics Consortium (PGC) $)^{24}\left(p=6.51 \times 10^{-14}\right.$ without the 23 andMe data). Although the PGC cross-disorder analysis used a subset of the samples included in the current study, these previously published OCD cases ${ }^{13}$ represent only $20 \%$ of the cases in the current analysis, and did not have the power to identify any individual locus with genome-wide significance for OCD alone ${ }^{13}$. Despite being under-powered in the crossdisorder analysis ${ }^{24}$, OCD was among only three of the eight phenotypes that were shown to contribute to the rs2581789 association results with m-values $>0.9$ ( $\mathrm{SCZ}$ and BP being the other two). These findings indicate that the genome-wide significant SNP in this study is not solely associated with OCD, but rather with multiple psychiatric phenotypes, reflecting a pleiotropic effect, possibly contributing to a shared underlying neurobiological susceptibility across a subset of psychiatric disorders and other neurobehavioral traits. These findings add to the confidence that this SNP, and the encompassing 3p21.1 region, is robustly, but not uniquely associated with OCD. Notably, another recent manuscript ${ }^{58}$, reported one genome-wide significant region for a compulsive disorder factor (rs9821797 on chromosome 3 p21.31, $\mathrm{P}=3.61 \times 10^{-9}, \mathrm{r}^{2}$ with rs $2581789<0.01$ and $\mathrm{D}$ $<0.2$ in European ancestry individuals), using structural equation modeling and summary statistics from 11 major psychiatric disorders. This locus reached a p-value of $4.32 \times 10^{-4}$ in the present OCD GWAS, although it should be noted that there was partial overlap between samples using the same sample as the PGC CDG study (see above).

Gene-based analyses, incorporating multiple types of functional annotations, including eQTLs, fetal and adult brain 
Hi-C information, and enrichment of tissue and single-cell RNAseq data, revealed no consistent associations. This is likely due to the absence of multiple, independent genomewide association loci from the primary GWAS, as these are typically necessary to have enough statistical power for such studies. However, two genes were identified by three of the four gene-based methods: the Transmembrane Protein 107 gene (TMEM107) and the CST Telomere Replication Complex Component 1 gene (CTC1), both located in the same locus on chromosome 17 (see Table 2 for the respective results of the four different gene-based methods). Given that SNPs in these genes were not identified as genome-wide significant in the primary GWAS meta-analysis, there is not yet sufficient evidence to consider the potential role(s) of genes or SNPs in these loci in the etiology of OCD.

Our analyses of the shared genetic risk between OCD and other psychiatric disorders provides deeper insights into the etiology of OCD. Previous work, including the crossdisorder analyses discussed above ${ }^{24 ; 58}$, have confirmed the shared genetic risk between OCD and two neuropsychiatric disorders that are highly co-morbid with OCD - TS ${ }^{12 ; 59}$ and $\mathrm{AN}^{60 ; 61}$. The above mentioned cross-disorder analyses also identified other psychiatric disorders as being correlated with OCD, albeit less strongly (SCZ, BP, and MDD). The present study, with its substantially increased sample size, confirms the genetic relationships between OCD and all of these disorders (TS, AN, SZ, BP, and MDD), and for the first time also shows significant genetic correlations between OCD and anxiety disorders, ADHD, as well as ASD (see Figure 4, Supplementary Figure S30 and Supplementary Tables S9 and S10).

In addition, our analyses of genome-wide genetic correlations between OCD and a wide range of additional brainand non-brain-based traits identified a number of significant results that warrant a more in-depth study in the future (see also below for potential limitations of our study). The significant negative association between genome-wide genetic risk for OCD and BMI, body-fat and hip circumference may be reflective of the shared genetic risk between OCD and AN, while the positive genetic correlation between OCD and tiredness, loneliness, neuroticism, and suicidality may represent the known clinical overlap of OCD, anxiety and MDD at a genetic level. Less clear regarding its interpretation is the significant genetic correlation between OCD and childhood maltreatment. Prior clinical studies have reported increased rates of childhood maltreatment in individuals with $\mathrm{OCD}^{62 ; 63}$, which may correlate with co-occurring depression and anxiety. However, further studies are needed to shed light on the genetic relationship and the overlap in genetic contributions to their etiology.

One potential study limitation is the fact that, due to potentially higher than normal rates of psychiatric co-morbidity in many of our OCD datasets (e.g. through inclusion of individuals diagnosed with more severe OCD), our results may be biased towards identifying genes that contribute to multiple comorbid psychiatric disorders, or alternatively to an underlying trait related to general psychopathology, rather than to OCD specifically. A substantial proportion of new samples in the current study were derived from large-scale biobanks and national registers, two of which were drawn from studies that originally aimed to study other psychiatric disorders, such as depression (AUS), or that included a higher-than-average number of cases with depression, ADHD, and ASD (iPSYCH). As such, there is a larger degree of heterogeneity in our meta-analysis which likely influenced the genetic correlation results. We conducted sensitivity analyses to address and to mitigate these potential biases by dividing the OCD meta-analysis into three subsets including a) only samples from large-scale biobanks, b) only clinical samples, and c) excluding the two studies with comorbid ascertainment (AUS and $\mathrm{PSYCH}$ ) from the analysis. We observed in part substantial changes in the point estimates of the genetic correlations with some disorders and traits (e.g. depression, anxiety disorders, and ADHD). We are currently conducting additional analyses to further address these limitations and will update the current version of this manuscript as soon as these results become available. Our observations echo similar results in other disorders (e.g. MDD) that call for more sophisticated analytical approaches (e.g. through inclusion of sensitivity meta-analytic methods based on structural equation modelling and other techniques) in order to combine samples with heterogeneous ascertainment. As with these other disorders the limitations inherent in our study design and in the available samples should be addressed in future genetic studies of OCD. In light of the above highlighted results, it is of note that we also performed leave-one-out polygenic risk score analyses using, among others, the previously published OCD meta-analysis of clinically-ascertained cases $^{13}$, which was used in all previous cross-disorder studies mentioned above. We found significant overlap in genetic liability between the prior OCD GWAS meta-analysis and leave-one-out GWAS data using predominantly samples that were newly added (mostly from large-scale biobanks) to our current meta-analysis (see Supplementary Figure S18). Once sample sizes sufficiently large to identify and replicate clear OCD susceptibility genes are collected, further analyses such as functional characterization, Mendelian randomization, and additional genetic correlation analyses may prove to be useful with the goal of further elucidating the underlying genetic etiology of OCD.

In sum, the present study provides insights into the current state of the largest GWAS for OCD to date. Our study identified the first definite genome-wide significant association of a locus with OCD (on chromosome 3p21.1). We are confident that with inclusion of additional samples (in the near future) we will be able to add new genome-wide significant regions to our current findings and further our understanding of OCD genetics. With these new meta-analysis results we will then also be able to use approaches (e.g., Mendelian Randomization and other) that will allow us to study the causal rela- 
medRxiv preprint doi: https://doi.org/10.1101/2021.10.13.21261078; this version posted October 23, 2021. The copyright holder for this preprint

(which was not certified by peer review) is the author/funder, who has granted medRxiv a license to display the preprint in perpetuity. It is made available under a CC-BY-NC-ND 4.0 International license.

tionship between the genetic underpinnings of closely related disorders.

\section{ACKNOWLEDGEMENTS}

A.A. and KJHV were supported by the Foundation Volksbond Rotterdam. MoBa is supported by the Norwegian Ministry of Health and Care Services and the Ministry of Education and Research. We are grateful to all the participating families in Norway who take part in this on-going cohort study. We thank the Norwegian Institute of Public Health for generating high-quality genomic data. This research is part of the HARVEST collaboration, supported by the Research Council of Norway (RCN) (229624). We also thank the NORMENT Centre for providing genotype data, funded by the RCN (223273), South East Norway Health Authority (SENHA) and KG Jebsen Stiftelsen. We further thank the Center for Diabetes Research, the University of Bergen for providing genotype data and performing quality control and imputation of the data funded by the ERC AdG project SELECTionPREDISPOSED, Stiftelsen Kristian Gerhard Jebsen, Trond Mohn Foundation, the NRC, the Novo Nordisk Foundation, the University of Bergen, and the Western Norway health Authorities. The Anorexia Nervosa Genetics Initiative (ANGI), an initiative of the Klarman Family Foundation. JK has been supported by the Academy of Finland (grant 336823). This study is funded by "Gentransmed" Centre of Excellence, ERDF nr 2014-2020.4.01.15-0012. MSA is a recipient of a Juan de la Cierva Incorporación contract (IJC2018-035346-I) from the Ministry of Science, Innovation and Universities, Spain. This investigation was supported by Instituto de Salud Carlos III (PI19/00721, P19/01224 and PI20/00041), and cofinanced by the European Regional Development Fund (ERDF), "la Marató de TV3" (092330/31), the Agència de Gestió d'Ajuts Universitaris i de Recerca-AGAUR, Generalitat de Catalunya (2014SGR1357 and 2017SGR1461) and the Pla estratègic de recerca i innovació en salut (PERIS), Generalitat de Catalunya (MENTAL-Cat; SLT006/17/287). This project has also received funding from the European Union's Horizon $2020 \mathrm{Re}$ search and Innovation Programme under the grant agreements No 667302 (CoCA) and 728018 (Eat2beNICE). J.A.R.Q was on the speakers' bureau and/or acted as consultant for Janssen-Cilag, Novartis, Shire, Takeda, Bial, Shionogi, Sincrolab, Novartis, BMS, Medice, Rubió, Uriach and Raffo in the last 3 years. He also received travel awards (air tickets + hotel) for taking part in psychiatric meetings from Janssen-Cilag, Rubió, Shire, Takeda, Shionogi, Bial and Medice. The Department of Psychiatry chaired by him received unrestricted educational and research support from the following companies in the last 3 years: Janssen- Cilag, Shire, Oryzon, Roche, Psious, and Rubió. Some of the data were collected as part of the following NIH grant: 1R01MH09338. The EPOC study was funded by the German Research Foundation, grants RA1971/8-1, WA731/10-1, WA731/15-1, and KA815/61. The GENOS study was supported by the German Research Foundation (GR 1912/1-1). The research at EstBB was supported by the European Union through the European Regional Development Fund (Project No. 2014-2020.4.01.16-0125), the Estonian Research Council grant PUT (PRG184), and through the CoMorMent project. CoMorMent has received funding from the European Union's Horizon 2020 Research and Innovation Programme under Grant agreement 847776. SEM is supported by an Australian NHMRC Investigator Grant (APP1172917). The AGDS was primarily funded by National Health and Medical Research Council (NHMRC) of Australia grant 1086683 . This work was further supported by NHMRC grants 1145645,1078901 and 1087889. LCC is supported by a QIMR Berghofer Institute fellowship. NORDiC is funded by NIMH R01 MH110427 (PI Crowley), NIMH R01 MH105500 (PI Crowley) and the Swedish Research Council grant 2015-02271 (P Mataix-Cols). Funding support for some of the Swedish controls was provided by the Klarman Family Foundation, the Swedish Research Council (Vetenskapsrådet, award: 538-2013-8864, and the NIMH Center for Collaborative Genomics Research on Mental Disorders (award U24 MH068457). This study was also supported by NIMH R01MH085321, NIMH R01MH101493, NIMH R01MH58376, and NIMH K20MH01065. It was furthermore funded by NIH R21 MH109938. Support was also provided by the German Research Foundation through grant KA815/6. The Research Council of Norway supported H. Ask, and T. Reichborn-Kjennerud (27611). A. Havdahl was supported by South East Norway Health Authority (2020022). Grant support was also provided from $\operatorname{RCN}(273291,262656,248778,223273)$ and the KG Jebsen Stiftelsen. LifeGene was supported by grants from the Ragnar and Torsten Söderberg Foundation, AFA Insurance, the Swedish Research Council and Karolinska Institutet. It is currently a core facility at Karolinska Institutet. The Trøndelag Health Study (HUNT) is a collaboration between HUNT Research Centre (Faculty of Medicine and Health Sciences, Norwegian University of Science and Technology NTNU), Trøndelag County Council, Central Norway Regional Health Authority, and the Norwegian Institute of Public Health. The genotyping was financed by the National Institute of health (NIH), University of Michigan, The Norwegian Research council, and Central Norway Regional Health Authority and the Faculty of Medicine and Health Sciences, Norwegian University of Science and Technology (NTNU). The genotype quality control and imputation has been conducted by the K.G. Jebsen center for genetic epidemiology, Department of public health and nursing, Faculty of medicine and health sciences, Norwegian University of Science and Technology (NTNU). EGOS was supported by a grant from the Beatrice and Samual A. Seaver Foundation to DEG. The OCD Collaborative Genetics Association Study (OCGAS) is a collaborative research study and was funded by the following NIMH Grant Numbers: MH071507 (G N), MH079489 (DAG), MH079487 (JM), MH079488 (AF), and MH079494 (JK). Yao Shugart and Wei Guo were also supported by the Intramural Research Program of the NIMH (MH002930-06).

The International Obsessive Compulsive Foundation Genetics Collaborative (IOCDF-GC) was supported by a grant from the David Judah Foundation (a private, non-industry related foundation established by a family affected by OCD), MH079489 (DLP), MH073250 (DLP), S40024 (JMS), MH 085057 (JMS), and
MH087748 (CAM).

We are deeply grateful for the participation of all subjects contributing to this research. In Sweden we would further like to thank the local collection team: Anders Juréus, Jessica Pege, Malin Rådström, Radja Satgunanthan-Dawoud, Milka Krestelica, and Birgitta Ohlander, as well as data manager Bozenna lliadou. We also wish to thank the National Quality Registry for Eating Disorders (RIKSÄT) for help with recruiting patients. We finally wish to thank the BBMRI.se and KI Biobank at Karolinska Institutet for professional biobank service. Data analysis for EstBB was partly carried out in the High-Performance Computing Center of University of Tartu. AGDS would like to thank all the people who helped in the conception, implementation, beta testing, media campaign and data cleaning. They would specifically like to acknowledge Dale Nyholt for advice on using the PBS for research; Ken Kendler, Patrick Sullivan, Andrew McIntosh and Cathryn Lewis for input on the questionnaire; Lorelle Nunn, Mary Ferguson, Lucy Winkler and Natalie Garden for data and sample collection; Natalia Zmicerevska, Alissa Nichles and Candace Brennan for participant recruitment support. Jonathan Davies, Luke Lowrey and Valeriano Antonini for support with IT aspects; Vera Morgan and Ken Kirkby for help with the media campaign. AGDS would like to thank VIVA! Communications for their effort in promoting the study. They also acknowledge David Whiteman and Catherine Olsen from QSkin. The authors thank the Psychiatric Genomics Consortium (PGC) for the use of their servers for data integration and analysis, the many families who have participated in the study, as well as the clinicians, study managers, and clinical interviewers at the respective study sites for their efforts in participant recruitment and clinical assessments. The views expressed here do not reflect the view of the National Institutes of Health, the Department of Health and Human Services, or the United States government.

\section{CONFLICTS OF INTEREST}

Ole Andreassen is a consultant to HealthLytix and has received speakers honorarium from Lundbeck and Sunovion in the past. CM Bulik reports: Shire (grant recipient, Scientific Advisory Board member); Idorsia (consultant); Lundbeckfonden (grant recipient); Pearson (author, royalty recipient). David Mataix-Cols receives royalties for contributing articles to UpToDate, Wolters Kluwer Health, and personal fees for editorial work from Elsevier, all unrelated to the current work. Hans-Joerg Grabe has received travel grants and speakers honoraria from Fresenius Medical Care, Neuraxpharm, Servier and Janssen Cilag, as well as research funding from Fresenius Medical Care. Jan Haavik has received lecture honoraria as part of continuing medical education programs sponsored by Shire, Takeda and Medice. Professor lan Hickie was an inaugural Commissioner on Australia's National Mental Health Commission (2012-18). He is the Co-Director, Health and Policy at the Brain and Mind Centre (BMC) University of Sydney, Australia. The BMC operates an early-intervention youth services at Camperdown under contract to headspace. Professor Hickie has previously led community-based and pharmaceutical industrysupported (Wyeth, Eli Lily, Servier, Pfizer, AstraZeneca) projects focused on the identification and better management of anxiety and depression. He was a member of the Medical Advisory Panel for Medibank Private until October 2017, a Board Member of Psychosis Australia Trust and a member of Veterans Mental Health Clinical Reference group. He is the Chief Scientific Advisor to, and a $5 \%$ equity shareholder in, InnoWell Pty Ltd. InnoWell was formed by the University of Sydney (45\% equity) and PwC (Australia; $45 \%$ equity) to deliver the $\$ 30 \mathrm{M}$ Australian Government-funded Project Synergy (2017-20; a three-year program for the transformation of mental health services) and to lead transformation of mental health services internationally through the use of innovative technologies. Erika Nurmi is member of the Scientific Advisory Board for Myriad Genetics and the Medical Advisory Board for Teva Pharmaceuticals and the Tourette Association of America.

\section{WEB RESOURCES}

1000G reference: www.internationalgenome.org/category/phase-3/

FUMA: https://fuma.ctglab.nl

ggplot2: www.ggplot2.tidyverse.org

HapMap3 reference: www.sanger.ac.uk/resources/downloads/human/hapmap3.html Single-cell RNAseq data: www.github.com/jbryois/scRNA_disease

\begin{abstract}
AFFILIATIONS
${ }^{1}$ Department of Psychology, Humboldt-Universität zu Berlin, Berlin, Germany 2 Departments of Psychiatry, Psychosomatics, and Psychotherapy, University Hospital Würzburg, Würzburg, Germany ${ }^{3}$ Department of Clinical Neuroscience, Karolinska Institutet, Stockholm, Sweden ${ }^{4}$ Department of Biomedicine, Aarhus University, Aarhus, Denmark ${ }^{5}$ Department of Psychiatry, Massachusetts General Hospital, Boston, MA, USA ${ }^{6}$ Stanley Center for Psychiatric Research, Broad Institute of MIT and Harvard, Cambridge, MA, USA ${ }^{7}$ Department of Mental Health, QIMR Berghofer Medical Research Institute, Brisbane, QLD, Australia ${ }^{8}$ Department of Genetics, University of North Carolina At Chapel Hill, Chapel Hill, NC, USA ${ }^{9}$ Department of Psychiatry, Amsterdam UMC, University of Amsterdam, Amsterdam, The Netherlands ${ }^{10}$ CIMUS (centre for Research In Molecular Medicine and Chronic Diseases), University of Santiago De Compostela, Santiago De Compostela, A Coruña, Spain ${ }^{11}$ Vanderbilt Genetics Institute, Vanderbilt University Medical Center, Nashville, TN, USA ${ }^{12}$ Departments of Psychiatry and Behavioral Sciences, SUNY Downstate Health Sciences University, Brooklyn, NY, USA ${ }^{13}$ Social, Genetic and Developmental Psychiatry Centre, King's College London, London, United Kingdom ${ }^{14}$ Departments of South London and Maudsley NHS Trust, NIHR Maudsley Biomedical Research Centre, London, United King-
\end{abstract}


dom ${ }^{15}$ Department of Psychiatry, Icahn School of Medicine At Mount Sinai, New York, NY, USA ${ }^{16}$ Departments of Medical Epidemiology and Biostatistics, Karolinska Institutet, Stockholm, Sweden ${ }^{17}$ Departments of Genetics and Computational Biology, QIMR Berghofer Medical Research Institute, Brisbane, QLD, Australia ${ }^{18}$ Departments of Psychiatry and Psychotherapy, University Hospital Bonn, Bonn, Germany ${ }^{19}$ Departments of Neurosciences and Mental Health, Hospital for Sick Children, Toronto, ON, Canada ${ }^{20}$ Department of Psychiatry, University Medical Center Utrecht, Utrecht, The Netherlands ${ }^{21}$ Second Opinion Outpatien Clinic, Ggnet, Warnsveld, The Netherlands ${ }^{22}$ Molecular Brain Science Department, Campbell Family Mental Health Research Institute, Centre for Addiction and Mental Health, Toronto, ON, Canada ${ }^{23}$ Department of Psychiatry, University of Toronto, Toronto, ON, Canada ${ }^{24}$ Waypoint Research Institute AND Outpatient Assessment and Treatment Services, Waypoint Centre for Mental Health Care, Penetanguishene, ON, Canada ${ }^{25}$ Department of Heath Sciences, University of Milano, Milano, Milano, Italy ${ }^{26}$ Departments of Child and Adolescent Psychiatry, Aarhus University Hospital, Psychiatry, Denmark, Aarhus University Hospital, Psychiatry, Aarhus, Denmark ${ }^{27}$ Institute of Clinical Medicine, Health, Aarhus University, Health, Aarhus University, Aarhus, Danmark ${ }^{28}$ Department of Neuropsychiatric Sciences, Università Vita-salute San Raffaele Milano Italy, Milano, Italy ${ }^{29}$ Departments of Psychiatry and Behavioral Sciences, Johns Hopkins University, Baltimore, MD, USA ${ }^{30}$ Departments of Roy J. and Lucille A. Carver College of Medicine, University of lowa, lowa City, IA, USA ${ }^{31}$ Child Study Center and Psychiatry, Yale University, New Haven, CT, USA ${ }^{32}$ Center for Psychiatric Research, Institution of Clinical Neuroscience, Stockholm, Sweden ${ }^{33}$ Department of MIND SCHOOLS, HOSPITAL SANT JOAN DE DEU, ESPLUGUES DE LLOBREGAT, BARCELONA, Spain ${ }^{34}$ Seaver Autism Center for Research and Treatment, Icahn School of Medicine At Moun Sinai, New York, NY, USA ${ }^{35}$ The Mindich Child Health and Development Institute, Icahn School of Medicine Mount Sinai, New York, NY, USA ${ }^{36}$ Department of Mclean Hospital, Harvard Medical School, Belmont, MA, USA ${ }^{37}$ Department of Psychiatry, Universidade De São Paulo, São Paulo, Brazil ${ }^{38}$ Department of Congenital Disorders, Statens Serum Institut, Copenhagen, Denmark ${ }^{39}$ Institute for Molecular Bioscience, University of Queensland, Brisbane, Queensland, Australia ${ }^{40}$ Child Health Research Centre, University of Queensland, Brisbane, QLD Australia ${ }^{41}$ Department of Pharmacogenetics, Instituto Nacional De Psiquitria Ramon De La Fuente Muñiz, Mexico City, Mexico ${ }^{42}$ Department of Surgery, Duke University, Durham, NC, USA ${ }^{43}$ Genomics Group, University of Santiago De Compostela, Santiago De Compostela, A Coruña, Spain ${ }^{44}$ Programa MIND Escoles, Hospital Sant Joan De Déu, ESPLUGUES DE LLOBREGAT, Barcelona, Spain ${ }^{45}$ Department of Departamento De Psiquiatría Y Medicina Legal, Universitat Autònoma De Barcelona, Bellaterra, Barcelona, Spain ${ }^{46}$ Department of Psychiatry, Ospedale San Raffaele, Milano, Italy ${ }^{47}$ Laboratory of Neuropsychiatry, IR CCS Santa Lucia Foundation, Rome, Italy ${ }^{48}$ Department of Psychiatry, University of Illinois At Chicago, Chicago, IL, USA ${ }^{49}$ Department of Psychiatry, University of Pittsburgh School of Medicine, Pittsburgh, PA, USA ${ }^{50}$ Department of Medical Ge netics, Oslo University Hospital, Oslo, Norway ${ }^{51}$ NORMENT, Clinical Science, University of Bergen, Bergen, Norway ${ }^{52}$ Departments of Genetics and Genome Biology, The Hospital for Sick Children, Toronto, ON, Canada ${ }^{53}$ Department of Genetics, University of Barcelona, Barcelona, Spain ${ }^{54}$ Department of Psychiatry, Mclean Hospital, Harvard Medical School, Belmont, MA, USA ${ }^{55}$ Departments of Public Health and Nursing, Norwegian University of Science and Technology, Trondheim Norway ${ }^{56}$ Biomedicine and The iSEQ Center, Aarhus University, Aarhus, Denmark ${ }^{57}$ The Lundbeck Foundation Initiative for Integrative Psychiatric Research, Ipsych, , Denmark ${ }^{58}$ National Institute of Mental Health, National Institutes of Health, Bethesda, MD, USA ${ }^{59}$ Genetic Epidemiology Research Branch, National Institute of Mental Health, National Institutes of Health, Bethesda, MD, Bethesda MD, USA ${ }^{60}$ Department of Biomedicine, University of Bergen, Bergen, Norway ${ }^{61}$ Department of Division of Psychiatry, Haukeland University Hospital, Bergen, Norway ${ }^{62}$ Department of Molde Hospital, Møre Og Romsdal Hospital Trust, Molde, Norway ${ }^{63}$ Department of Mental Health, Norwegian University of Science and Technology, Trondheim, Norway ${ }^{64}$ Department of Mental Disorders, Norwegian Institute of Public Health, Oslo, Norway ${ }^{65}$ Nic Waals Institute, Lovisenberg Diaconal Hospital, Oslo, Norway ${ }^{66}$ PROMENTA Research Center, Psychology, University of Oslo, Oslo, Norway ${ }^{67}$ Department of Medicine, Vanderbilt University Medical Center, Nashville, TN, USA ${ }^{68}$ Department of Psychiatry, Massachusetts General Hos pital and Harvard Medical School, Boston, MA, USA ${ }^{69}$ Departments of Bioinformatics and Integrative Biology, University of Massachusetts Medical School, Worces ter, MA, USA ${ }^{70}$ Department of Vertebrate Genomics, Broad Institute of MIT and Harvard, Cambridge, MA, USA ${ }^{71}$ Department of Psychiatry, University of Pittsburgh, Pittsburgh, PA, USA ${ }^{72}$ Department of Psychiatry, Johns Hopkins University, Baltimore, MD, USA ${ }^{73}$ Institute of Genomics, University of Tartu, Tartu, Estonia ${ }^{74}$ Carracci Medical Group, Mexico City, Mexico ${ }^{75}$ Departments of Psychiatry and Human Behavior, University of Californiairvine (UCI), Irvine, CA, USA ${ }^{76}$ Department of Mental Health, Johns Hopkins Bloomberg School of Public Health Baltimore, MD, USA ${ }^{77}$ COBRE Center for Neuromodulation, Butler Hospital, Providence, RI, USA ${ }^{78}$ Departments of Psychiatry and Human Behavior, Alpert Medical School, Brown University, Providence, RI, USA ${ }^{79}$ Department of Psychiatry, Dalhousie University, Halifax, NS, Canada ${ }^{80}$ Departments of Psychiatry and Biobehavioral Sciences, University of California, Los Angeles, Los Angeles, CA USA ${ }^{81}$ NORMENT Center of Excellence (coe), Institute of Clinical Medicine, University of Oslo, Oslo, Norway ${ }^{82}$ Departments of Division of Mental Health and Addiction, Oslo University Hospital, Oslo, Norway ${ }^{83}$ Psychiatric and Neurodevelopmental Genetics Unit, Harvard Medical School, Boston, MA, USA ${ }^{84}$ Institute for Molecular Medicine Finland - FIMM, University of Helsinki, Helsinki, Finland ${ }^{85}$ Departments of Psychiatry and Psychotherapy, University of Cologne, Cologne Germany ${ }^{86}$ Departments of Neurodegenerative Diseases and Geriatric Psychiatry, University of Bonn, Bonn, Germany ${ }^{87}$ DZNE Bonn, German Center for Neu- rodegenerative Diseases (DZNE), Bonn, Germany ${ }^{88}$ Psychiatry and Glenn Biggs Institute for Alzheimer's and Neurodegenerative Diseases, UT Health San Antonio, San Antonio, TX, USA ${ }^{89}$ Department of Mental Disorders, Norwegian Institute of Public Health, New York, NY, USA ${ }^{90}$ Department of Medicine, Massachusetts General Hospital and Harvard Medical School, Boston, MA, USA ${ }^{91}$ Departments of Psychaitry and Psychotherapy, Charité Universitätsmedizin, Berlin, Germany 92 Department of University Health Careservices - SMURB, Federal University of Bahia, Salvador, Brazil ${ }^{93}$ Departments of Psychiatry and Psychotherapy, Medical Center - University of Freiburg, Freiburg, Germany ${ }^{94}$ Department of Psychiatry, Amsterdam UMC Location Vumc, Amsterdam, The Netherlands ${ }^{95}$ Departments of Psychiatry and The Behavioral Sciences, Keck School of Medicine of USC, Los Angeles, CA, USA ${ }^{96}$ Psychiatric Genetics Unit, Group of Psychiatry, Mental Health and Addiction, Vall D'hebron Research Institute (VHIR), Barcelona, Spain ${ }^{97}$ Department of Psychiatry, Hospital Universitari Vall D'hebron, Barcelona, Spain ${ }^{98}$ Department of Instituto De Salud Carlos III, Biomedical Network Research Centre On Mental Health (CIBERSAM), Madrid, Spain ${ }^{99}$ Department of Biology, Universitat De Barcelona, Barcelona, Spain ${ }^{100}$ Departments of Clinical and Molecular Medicine, Norwegian University of Science and Technology, Trondheim, Norway ${ }^{101}$ K. G. Jebsen Center for Genetic Epidemiology, Norwegian University of Science and Technology, Trondheim, Norway ${ }^{102}$ Departments of Molecular Medicine and Surgery, Karolinska Institutet, Stockholm, Sweden ${ }^{103}$ Departments of Psychiatry, Pediatrics, and Pharmacology, Vanderbilt University Medical Center, Nashville, TN, USA ${ }^{104}$ Department of Precision Medicine, City of Hope, Monrovia, CA, USA ${ }^{105}$ Laboratory of Clinical Science, NIMH Intramural Research Program, Bethesda MD, USA ${ }^{106}$ Departments of Research, Innovation and Education, Oslo University Hospital, Oslo, Norway ${ }^{107}$ Departments of Medicine and Health Sciences, Norwegian University of Science and Technology, Trondheim, Norway ${ }^{108}$ Department of Neurology, Oslo University Hospital, Oslo, Norway ${ }^{109}$ Department of Computional Biology,, Fudan University., Fudan, PRC ${ }^{110}$ Department of Bellvitge University, University of Barcelona, Barcelona, Spain ${ }^{111}$ Psychosomatic Department, Windach Hospital of Neurobehavioural Research and Therapy, Windach, Germany ${ }^{112}$ Department of Psychiatry, University of North Carolina At Chapel Hill, Chapel Hill, NC, USA ${ }^{113}$ Department of Nutrition, University of North Carolina At Chapel Hill, Chapel Hill, NC, USA ${ }^{114}$ Departments of Rijksuniversiteit Groningen and Psychiatry, University Medical Center Groninge, Groningen, The Netherlands ${ }^{115}$ Department of Specialized Training, Drenthe Mental Health Care Institute, , ${ }^{116}$ Institute of Biomedical Technologies, Italian National Centre for Research, Segrate, Milano, Italy ${ }^{117}$ Child and Adolesccent Psycchiatry Department, APHP, Paris, France ${ }^{118}$ Department of Psychiatry, Institute of The Royalnetherlands Academy of Arts and Sciences (NIN-KNAW), Amsterdam, The Netherlands ${ }^{119}$ Department of Medicine, University of New South Wales, Randwick, NSW Australia ${ }^{120}$ Departments of Psychiatry and Psychotherapy, University of Göttingen, Göttingen, Germany ${ }^{121}$ Department of Psychiatry, New York State Psychiatric Institute, New York, NY, USA ${ }^{122}$ Department of Psychiatry, Columbia University Medical Center, New York, NY, USA ${ }^{123}$ Department of Psychiatry, Harvard Medical School, Boston, MA, USA ${ }^{124}$ Departments of Psychiatry and Psychotherapy, University Medicine Greifswald, Greifswald, Greifswald ${ }^{125}$ RRD Center for Neurorestoration and Neurotechnology, Providence VA Medical Center, Providence, RI ${ }^{126}$ Department of Psychiatry, University of Michigan, Ann Arbor, MI, USA ${ }^{127}$ Brain and Mind Center, The University of Sydney, Sydney, NSW, Australia ${ }^{128}$ Department of Biostatistics, Johns Hopkins Bloomberg School of Public Health, Baltimore, MD, USA ${ }^{129}$ Institute of Population Health Sciences, National Health Research Institutes, , Taiwan ${ }^{130}$ Institute of Neuroscience and Physiology, University of Gothenburg, Gothenburg, Sweden ${ }^{131}$ Bergen Center for Brain Plasticity, Haukeland University Hospital, Bergen, Norway ${ }^{132}$ Department of Clinical Science, University of Bergen, Bergen, Norway ${ }^{133}$ Departments of Addictology and Psychiatry, Univ Paris Est Créteil, AP-HP, Inserm, Paris, France ${ }^{134}$ Department of Psychiatry, Stellenbosch University, Stellenbosch, South Africa ${ }^{135}$ National Centre for Register-based Research, Aarhus University, Aarhus, Denmark ${ }^{136}$ Centre for Integrated Register-based Research, Aarhus University, Aarhus, Denmark ${ }^{137}$ Analytic and Translational Genetics Unit, Massachusetts General Hospital, Boston, MA, USA ${ }^{138}$ Program In Medical and Population Genetics, Broad Institute of Harvard and MIT, Cambridge, MA, USA ${ }^{139}$ Department of Psychiatric Genetics, Grupo Médico Carracci INMEGEN, Mexico City, México ${ }^{140}$ Department of Psychiatric Genetics, Instituto Nacional De Medicina Genómica, México City, Mexico ${ }^{141}$ CORE - Copenhagen Research Center for Mental Health, Copenhagen University Hospital, Copenhagen, Denmark ${ }^{142}$ Department of Clinical Medicine, University of Copenhagen, Copenhagen, Denmark ${ }^{143}$ Department of Psychiatry, Rutgers University, Piscataway, NJ, USA ${ }^{144}$ Department of Psychiatry, Harvard University, Boston, MA, USA ${ }^{145}$ Department of Psychiatry, Yale University, New Haven, CT, USA ${ }^{146}$ Department of Clinical Genetics, VU University Medical Center Amsterdam, Amsterdam, The Netherlands ${ }^{147}$ Department of Psychiatry, Hospital Universitari Vall D'hebron, Barcelona, Catalonia, Spain ${ }^{148}$ Group of Psychiatry, Mental Health and Addictions, Vall D'hebron Research Institute (VHIR), Barcelona, Spain ${ }^{149}$ Group 27, Biomedical Network Research Centre On Mental Health (CIBERSAM), Barcelona, Spain ${ }^{150}$ Departments of Psychiatry and Forensic Medicine, Universitat Autònoma De Barcelona, Barcelona, Spain ${ }^{151}$ Department of Psychiatry, Sunnybrook Health Sciences Centre, , ${ }^{152}$ Department of Psychiatry, Federal University of São Paulo (UNIFESP), São Paulo, Brazil ${ }^{153}$ Departments of Psychiatry and Behavioral Neurosciences, Wayne State University School of Medicine, Detroit, MI, USA ${ }^{154}$ Departments of Psychiatry and Behavioral Sciences, Baylor College of Medicine, Houston, TX, USA ${ }^{155}$ Psychiatry and Neuroscience Institute, University of Cape Town, Cape Town, Western Cape, South Africa ${ }^{156}$ Department of Psychiatry, University of British Columbia, Vancouver, BC, Canada ${ }^{157}$ Department of Pharmacology, Northwestern University Feinberg School of Medicine, Chicago, IL, USA ${ }^{158}$ Center for Genetic Medicine, Northwestern University Feinberg School of 
medRxiv preprint doi: https://doi.org/10.1101/2021.10.13.21261078; this version posted October 23, 2021. The copyright holder for this preprint

(which was not certified by peer review) is the author/funder, who has granted medRxiv a license to display the preprint in perpetuity.

\author{
It is made available under a CC-BY-NC-ND 4.0 International license .
}

Medicine, Chicago, IL, USA ${ }^{159}$ Department of Cardiology, University of Milan, Milan, Italy ${ }^{160}$ Department of Mental Health Services, Copenhagen University Hospital, Copenhagen, Denmark ${ }^{161}$ GLOBE Institute, University of Copenhagen, Copenhagen, Denmark ${ }^{162}$ Institute of Clinical Medicine, University of Oslo, Oslo, Norway ${ }^{163}$ Departments of Child and Adolescent Psychiatry and Psychotherapy, University of Zurich, Zürich, Switzerland ${ }^{164}$ Neuroscience Center Zurich, University of Zurich and The ETH Zuric, Zurich, Switzerland ${ }^{165}$ Zurich Center for Integrative Human Physiology, University of Zurich, Zurich, Switzerland ${ }^{166}$ Department of Psychology, University of Bergen, Bergen, Norway ${ }^{167}$ Psychosis Reasearch Unit, Aarhus University Hospital - Psychiatry, 8200 Aarhus N, Denmark ${ }^{168}$ Psychiatric Genetics Unit, Vall D'hebron Research Institute (VHIR), Universitat Autònoma De Barcelona, Barcelona, Spain ${ }^{169}$ Biomedical Network Research Centre On Mental Health (CIBERSAM), Barcelona, Spain. 3 Biomedical Network Research Centre On Mental Health (CIBERSAM), Instituto De Salud Carlos III, Madrid, Spain ${ }^{170}$ Departments of Genetics, Microbiology and Statistics, University of Barcelona, Barcelona, Spain ${ }^{171}$ Center for Basics In Neuromodulation, University of Freiburg, Freiburg, Germany ${ }^{172}$ Department of Medicine, University of Oslo, Oslo, Norway ${ }^{173}$ Helsinki Institute of Life Science, University of Helsinki, Helsinki, Finland ${ }^{174}$ Department of Psychiatry, Cumming School of Medicine, University of Calgary, Calgary, AB, Canada ${ }^{175}$ Program In Genetics and Genome Biology, Hospital for Sick Children, Toronto, ON, Canada ${ }^{176}$ Department of Cell Biology, SUNY Downstate Health Sciences University, Brooklyn, NY, USA ${ }^{177}$ Department of Psychiatry, Amsterdam UMC Location AMC, Amsterdam, The Netherlands ${ }^{178}$ Psychiatry and Genetics Institute, Center for OCD, Anxiety and Related Disorders, University of Florida, Gainesville, FL, USA ${ }^{179}$ Department of Psychiatry, Center for Genomic Medicine, Massachusetts General Hospital, Harvard Medical School, Boston, MA, USA ${ }^{180}$ Departments of Psychiatry and Community Health and Epidemiology, Dalhousie University, Halifax, NS, Canada ${ }^{181}$ Institute of Psychiatric Phenomics and Genomics (IPPG), University Hospital of Munich, Munich, Germany ${ }^{182}$ Contributed equally ${ }^{183}$ Contributed equally 


\section{References}

1. Ruscio AM, Stein DJ, Chiu WT, Kessler RC. The epidemiology of obsessive-compulsive disorder in the National Comorbidity Survey Replication. Molecular Psychiatry. 2010;15(1)

2. Stein DJ, Costa DLC, Lochner C, Miguel EC, Reddy YCJ, Shavitt RG, et al.. Obsessive-compulsive disorder. Nature Publishing Group; 2019. Available from: www . nature. $\mathrm{com} / \mathrm{nrdp}$.

3. Teixeira J, Carvalho I, Pires P. $706-110$ years after first report of obsessive compulsive disorder in childhood - where do we stand? European Psychiatry. 2013;28.

4. Anholt GE, Aderka IM, Van Balkom AJLM, Smit JH, Schruers K, Van Der Wee NJA, et al. Age of onset in obsessive-compulsive disorder: Admixture analysis with a large sample. Psychological Medicine. 2014;44(1).

5. Lochner C, Fineberg NA, Zohar J, Van Ameringen M, Juven-Wetzler A, Altamura AC, et al. Comorbidity in obsessive-compulsive disorder (OCD): A report from the International College of Obsessive-Compulsive Spectrum Disorders (ICOCS). Comprehensive Psychiatry. 2014 10;55(7):1513-1519.

6. Browne HA, Gair SL, Scharf JM, Grice DE. Genetics of obsessive-compulsive disorder and related disorders. W.B. Saunders; 2014. Available from: / pmc/articles/ PMC4143777/?report=abstracthttps://ww.ncbi.nlm.nih.gov/pmc/ articles/PMC4143777/.

7. Simpson HB, Van Den Heuvel OA, Miguel EC, Reddy YCJ, Stein DJ, Lewis-Fernández R, et al. Toward identifying reproducible brain signatures of obsessive-compulsive profiles: Rationale and methods for a new global initiative. BMC Psychiatry. 2020 2;20(1). Available from: /pmc/articles/PMC7023814/ ?report=abstracthttps://www.ncbi. nlm.nih.gov/pmc/articles/PMC7023814/.

8. Mataix-Cols D, Do Rosario-Campos MC, Leckman JF. A multidimensional model of obsessive-compulsive disorder; 2005

9. Pauls DL. The genetics of obsessive compulsive disorder: A review of the evidence. American Journal of Medical Genetics Part C: Seminars in Medical Genetics. 2008 5;148C(2):133-139. Available from: http://doi.wiley.com/10.1002/ajmg.c. 30168.

10. van Grootheest DS, Cath DC, Beekman AT, Boomsma DI. Twin Studies on Obsessive-Compulsive Disorder: A Review. Twin Research and Human Genetics. 2005 10;8(5):450-458. Available from: http://journals. cambridge.org/abstract_ S1832427400005648.

11. Burton CL, Park LS, Corfield EC, Forget-Dubois N, Dupuis A, Sinopoli VM, et al. Heritability of obsessive-compulsive trait dimensions in youth from the general population. Translational Psychiatry. 2018;8(1)

12. Davis LK, Yu D, Keenan CL, Gamazon ER, Konkashbaev Al, Derks EM, et al. Partitioning the Heritability of Tourette Syndrome and Obsessive Compulsive Disorder Reveals Differences in Genetic Architecture. PLoS Genetics. 2013;9(10).

13. Arnold PD, Askland KD, Barlassina C, Bellodi L, Bienvenu OJ, Black D, et al. Revealing the complex genetic architecture of obsessive-compulsive disorder using meta-analysis. Molecular Psychiatry. 2018 5;23(5):1181-1188.

14. Cappi C, Oliphant ME, Péter Z, Zai G, Conceição do Rosário M, Sullivan CAW, et al. De Novo Damaging DNA Coding Mutations Are Associated With Obsessive-Compulsive Disorder and Overlap With Tourette's Disorder and Autism. Biological Psychiatry. 2020 6;87(12):1035-1044. Available from: https://pubmed.ncbi.nlm.nih.gov/ $31771860 \%$

15. McGrath LM, Yu D, Marshall C, Davis LK, Thiruvahindrapuram B, Li B, et al. Copy number variation in obsessive-compulsive disorder and tourette syndrome: A cross-disorder study. Journal of the American Academy of Child and Adolescent Psychiatry. 2014;53(8):910-919. Available from: https://pubmed.ncbi.nlm.nih.gov/25062598/.

16. Gazzellone MJ, Zarrei M, Burton CL, Walker S, Uddin M, Shaheen SM, et al. Uncovering obsessive-compulsive disorder risk genes in a pediatric cohort by high-resolution analysis of copy number variation. Journal of Neurodevelopmental Disorders. 2016;8(1).

17. Grünblatt E, Oneda B, Ekici AB, Ball J, Geissler J, Uebe S, et al. High resolution chromosomal microarray analysis in paediatric obsessive-compulsive disorder. BMC Medical Genomics. 2017 11;10(1):1-11. Available from: https://bmcmedgenomics . biomedcentral.com/articles/10.1186/s12920-017-0299-5.

18. Stewart SE, Yu D, Scharf JM, Neale BM, Fagerness JA, Mathews CA, et al. Genomewide association study of obsessive-compulsive disorder. Molecular Psychiatry. 2013 7;18(7):788-798.

19. Mattheisen M, Samuels JF, Wang Y, Greenberg BD, Fyer AJ, Mccracken JT, et al. Genomewide association study in obsessive-compulsive disorder: Results from the OCGAS. Molecular Psychiatry. 2015 3;20(3):337-344.

20. Sullivan PF, Agrawal A, Bulik CM, Andreassen OA, Børglum AD, Breen G, et al. Psychiatric genomics: An update and an Agenda. American Journal of Psychiatry. 2018 1;175(1):1527. Available from: https://pubmed.ncbi.nlm.nih.gov/28969442/.

21. Howard DM, Adams MJ, Clarke TK, Hafferty JD, Gibson J, Shirali M, et al. Genome-wide meta-analysis of depression identifies 102 independent variants and highlights the importance of the prefrontal brain regions. Nature Neuroscience. 2019;22(3).

22. Smoller JW, Andreassen OA, Edenberg HJ, Faraone SV, Glatt SJ, Kendler KS. Psychiatric genetics and the structure of psychopathology; 2019

23. Ripke S, Neale BM, Corvin A, Walters JTR, Farh KH, Holmans PA, et al. Biological insights from 108 schizophrenia-associated genetic loci. Nature. 2014;511(7510).

24. Lee PH, Anttila V, Won H, Feng YCA, Rosenthal J, Zhu Z, et al. Genomic Relationships, Novel Loci, and Pleiotropic Mechanisms across Eight Psychiatric Disorders. Cell. 2019 12;179(7):1469-1482.

25. Watson HJ, Yilmaz Z, Thornton LM, Hübel C, Coleman JRI, Gaspar HA, et al. Genomewide association study identifies eight risk loci and implicates metabo-psychiatric origins for anorexia nervosa. Nature Genetics. 2019 8;51(8):1207-1214. Available from: https : //doi.org/10.1038/s41588-019-0439-2.

26. American Psychiatric Association. Diagnostic and statistical manual of mental disorders (4th ed., Text Revision). 2000;
27. McCarthy S, Das S, Kretzschmar W, Delaneau O, Wood AR, Teumer A, et al. A reference panel of 64,976 haplotypes for genotype imputation. Nature Genetics. 2016 10;48(10):1279-1283

28. Auton A, Abecasis GR, Altshuler DM, Durbin RM, Bentley DR, Chakravarti A, et al.. A global reference for human genetic variation; 2015.

29. Lam M, Awasthi S, Watson HJ, Goldstein J, Panagiotaropoulou G, Trubetskoy V, et al. RICOPILI: Rapid Imputation for COnsortias PIpeLIne. Bioinformatics. 2020 2;36(3):930-933. Available from: https://pubmed.ncbi.nlm.nih.gov/31393554/.

30. Willer CJ, Li Y, Abecasis GR. METAL: Fast and efficient meta-analysis of genomewide association scans. Bioinformatics. 2010;26(17).

31. de Leeuw CA, Mooij JM, Heskes T, Posthuma D. MAGMA: Generalized Gene-Set Analysis of GWAS Data. PLoS Computational Biology. 2015 4;11(4). Available from: https:// pubmed.ncbi.nlm.nih.gov/25885710/.

32. Gerring ZF, Gamazon ER, Derks EM. A gene co-expression network-based analysis of multiple brain tissues reveals novel genes and molecular pathways underlying major depression. PLoS Genetics. 2019 7;15(7). Available from: https ://pubmed.ncbi.nlm. nih.gov/31306407/.

33. Gamazon ER, Wheeler HE, Shah KP, Mozaffari SV, Aquino-Michaels K, Carroll RJ, et al A gene-based association method for mapping traits using reference transcriptome data. Nature Genetics. 2015 8:47(9):1091-1098. Available from: https://pubmed.ncbi. nlm.nih.gov/26258848/.

34. Consortium TG. The GTEx Consortium atlas of genetic regulatory effects across human tissues. Science (New York, NY). 2020 9;369(6509):1318-1330. Available from: http: //science, sciencemag. org/.

35. Sey NYA, Hu B, Mah W, Fauni H, McAfee JC, Rajarajan P, et al. A computational tool (H MAGMA) for improved prediction of brain-disorder risk genes by incorporating brain chromatin interaction profiles. Nature Neuroscience. 2020 4;23(4):583-593. Available from: https://pubmed.ncbi.nlm.nih.gov/32152537/.

36. Watanabe K, Taskesen E, Van Bochoven A, Posthuma D. Functional mapping and annotation of genetic associations with FUMA. Nature Communications. 2017 12;8(1). Available from: https: //pubmed,ncbi,nlm.nih.gov/29184056/.

37. Li M, Santpere G, Kawasawa YI, Evgrafov OV, Gulden FO, Pochareddy S, et al. Integrative functional genomic analysis of human brain development and neuropsychiatric risks. Science. 2018;362(6420)

38. Bryois J, Skene NG, Hansen TF, Kogelman LJA, Watson HJ, Liu Z, et al. Genetic identification of cell types underlying brain complex traits yields insights into the etiology of Parkinson's disease. Nature Genetics. 2020 5;52(5):482-493. Available from: https: //pubmed.ncbi.nlm.nih.gov/32341526/.

39. Aguet F, Brown AA, Castel SE, Davis JR, He Y, Jo B, et al. Genetic effects on gene expression across human tissues. Nature. 2017 10;550(7675):204-213. Available from: https://pubmed.ncbi.nlm.nih.gov/29022597/.

40. Zeisel A, Hochgerner H, Lönnerberg P, Johnsson A, Memic F, van der Zwan J, et al. Molecular Architecture of the Mouse Nervous System. Cell. 2018 8;174(4):999-1014. Available from: https://pubmed.ncbi.nlm.nih.gov/30096314/.

41. Bulik-Sullivan B, Loh PR, Finucane HK, Ripke S, Yang J, Patterson N, et al. LD score regression distinguishes confounding from polygenicity in genome-wide association studies. Nature Genetics. 2015 2;47(3):291-295.

42. Wickham H. ggplot2: Elegant Graphics for Data Analysis. Springer-Verlag New York; 2016. Available from: https://ggplot2.tidyverse.org.

43. Wang D, Liu S, Warrell J, Won H, Shi X, Navarro FCP, et al. Comprehensive functiona genomic resource and integrative model for the human brain. Science. 2018 12;362(6420). Available from: https://pubmed.ncbi.nlm.nih.gov/30545857/.

44. Giusti-Rodríguez P, Lu L, Yang Y, Crowley CA, Liu X, Juric I, et al. Using three-dimensional regulatory chromatin interactions from adult and fetal cortex to interpret genetic results for psychiatric disorders and cognitive traits. bioRxiv. 2018 1;18(8):20. Available from: http: /dx.doi.org/10.1101/406330.

45. Bulik-Sullivan B, Finucane HK, Anttila V, Gusev A, Day FR, Loh PR, et al. An atlas of genetic correlations across human diseases and traits. Nature Genetics. 2015 11;47(11):12361241. Available from: https://pubmed.ncbi.nlm.nih.gov/26414676/.

46. Finucane HK, Bulik-Sullivan B, Gusev A, Trynka G, Reshef Y, Loh PR, et al. Partitioning heritability by functional annotation using genome-wide association summary statistics. Nature Genetics. 2015 11;47(11):1228-1235. Available from: https://pubmed.ncbi.nlm. nih.gov/26414678/.

47. Pardiñas AF, Holmans P, Pocklington AJ, Escott-Price V, Ripke S, Carrera N, et al. Common schizophrenia alleles are enriched in mutation-intolerant genes and in regions under strong background selection. Nature Genetics. 2018 3;50(3):381-389. Available from: https: //pubmed.ncbi.nlm.nih.gov/29483656/.

48. Ruderfer DM, Ripke S, McQuillin A, Boocock J, Stahl EA, Pavlides JMW, et al. Genomic Dissection of Bipolar Disorder and Schizophrenia, Including 28 Subphenotypes. Cell. 2018 6;173(7):1705-1715. Available from: https://pubmed.ncbi.nlm.nih.gov/ 29906448/.

49. Pulit SL, Stoneman C, Morris AP, Wood AR, Glastonbury CA, Tyrrell J, et al. Meta-Analysis of genome-wide association studies for body fat distribution in 694649 individuals of European ancestry. Human Molecular Genetics. 2019;28(1).

50. Nagel M, Watanabe K, Stringer S, Posthuma D, Van Der Sluis S. Item-level analyses reveal genetic heterogeneity in neuroticism. Nature Communications. 2018 12;9(1). Available from: https://pubmed.ncbi.nlm.nih.gov/29500382/.

51. Nagel M, Jansen PR, Stringer S, Watanabe K, De Leeuw CA, Bryois J, et al. Metaanalysis of genome-wide association studies for neuroticism in 449,484 individuals identifies novel genetic loci and pathways. Nature Genetics. 2018 7;50(7):920-927. Available from: https://doi.org/10.1038/s41588-018-0151-7.

52. Watanabe K, Stringer S, Frei O, Umićević Mirkov M, de Leeuw C, Polderman TJC, et al. A global overview of pleiotropy and genetic architecture in complex traits. Nature Genetics. 2019 9;51(9):1339-1348. Available from: https://pubmed.ncbi.nlm.nih. gov/31427789/.

53. Baselmans BML, Jansen R, Ip HF, van Dongen J, Abdellaoui A, van de Weijer MP, 
medRxiv preprint doi: https://doi.org/10.1101/2021.10.13.21261078; this version posted October 23, 2021. The copyright holder for this preprint (which was not certified by peer review) is the author/funder, who has granted medRxiv a license to display the preprint in perpetuity.

\section{It is made available under a CC-BY-NC-ND 4.0 International license .}

et al. Multivariate genome-wide analyses of the well-being spectrum. Nature Genetics. 2019 3;51(3):445-451. Available from: https://pubmed.ncbi.nlm.nih.gov/ $30643256 /$.

54. Hill WD, Weiss A, Liewald DC, Davies G, Porteous DJ, Hayward C, et al. Genetic contributions to two special factors of neuroticism are associated with affluence, higher intelligence, better health, and longer life. Molecular Psychiatry. 2020 11;25(11):3034-3052. Available from: https://pubmed.ncbi.nlm.nih.gov/30867560/.

55. Luciano M, Hagenaars SP, Davies G, Hill WD, Clarke TK, Shirali M, et al. Association analysis in over 329,000 individuals identifies 116 independent variants influencing neuroticism. Nature Genetics. 2018 1;50(1):6-11. Available from: https://pubmed.ncbi. nlm.nih.gov/29255261/.

56. Lee JJ, Wedow R, Okbay A, Kong E, Maghzian O, Zacher M, et al. Gene discovery and polygenic prediction from a genome-wide association study of educational attainment in 1.1 million individuals. Nature Genetics. 2018 8;50(8):1112-1121. Available from: https: //pubmed.ncbi.nlm.nih.gov/30038396/.

57. Wang C, Zhan X, Bragg-Gresham J, Kang HM, Stambolian D, Chew EY, et al. Ancestry estimation and control of population stratification for sequence-based association studies. Nature Genetics. 2014;46(4):409-415.

58. Grotzinger AD, Mallard TT, Akingbuwa WA, Ip HF, Adams MJ, Lewis CM, et al. Genetic Architecture of 11 Major Psychiatric Disorders at Biobehavioral, Functional Genomic, and Molecular Genetic Levels of Analysis. medRxiv. 2020;.

59. Yu D, Sul JH, Tsetsos F, Nawaz MS, Huang AY, Zelaya I, et al. Interrogating the genetic determinants of Tourette's syndrome and other tiC disorders through genome-wide association studies. American Journal of Psychiatry. 2019 3;176(3):217-227. Available from: /pmc/articles/PMC6677250/?report=abstracthttps://www.ncbi. nlm.nih.gov/pmc/articles/PMC6677250/.

60. Anttila V, Bulik-Sullivan B, Finucane HK, Walters RK, Bras J, Duncan L, et al. Analysis of shared heritability in common disorders of the brain. Science. 2018 6;360(6395). Available from: https://pubmed.ncbi.nlm.nih.gov/29930110/.

61. Yilmaz Z, Halvorsen M, Bryois J, Yu D, Thornton LM, Zerwas S, et al. Examination of the shared genetic basis of anorexia nervosa and obsessive-compulsive disorder. Molecular Psychiatry. 2020;25(9).

62. Mathews CA, Kaur N, Stein MB. Childhood trauma and obsessive-compulsive symptoms. Depression and Anxiety. 2008 9;25(9):742-751. Available from: http://doi.wiley. $\mathrm{com} / 10.1002 / \mathrm{da} .20316$.

63. Osland S, Arnold PD, Pringsheim T. The prevalence of diagnosed obsessive compulsive disorder and associated comorbidities: A population-based Canadian study. Psychiatry Research. 2018 10;268:137-142. Available from: https://pubmed.ncbi.nlm.nih. gov/30025284/. 
medRxiv preprint doi: https://doi.org/10.1101/2021.10.13.21261078; this version posted October 23, 2021. The copyright holder for this preprint (which was not certified by peer review) is the author/funder, who has granted medRxiv a license to display the preprint in perpetuity. It is made available under a CC-BY-NC-ND 4.0 International license.

\section{Figures and Tables}


medRxiv preprint doi: https://doi.org/10.1101/2021.10.13.21261078; this version posted October 23, 2021. The copyright holder for this preprint

(which was not certified by peer review) is the author/funder, who has granted medRxiv a license to display the preprint in perpetuity.

It is made available under a CC-BY-NC-ND 4.0 International license.

Table 1. LD-independent genomic regions with $p<1 \times 10^{-6}$ in the OCD meta-analysis and their associated genes

\begin{tabular}{|c|c|c|c|c|c|c|c|c|c|c|}
\hline SNP & CHR & BP & $\mathbf{P}$ & OR & SE & A1/A2 & FRQ_A & FRQ_U & INFO & genes.6.50kb(dist2index) \\
\hline rs2581789 & 3 & 53096230 & $2.138 \times 10^{-8}$ & 0.91677 & 0.0155 & $\mathrm{C} / \mathrm{T}$ & 0.328 & 0.353 & 0.996 & $\begin{array}{l}\text { NEK4(-241.3),ITIH1(-220.2), } \\
\text { ITIH3(-203.2),ITIH4(-181.5), } \\
\text { ITIH4-AS1(-186.9),MUSTN1(-177.2), } \\
\text { STIMATE-MUSTN1(-114.7), } \\
\text { STIMATE(-114.7),MIR8064(-165.7), } \\
\text { SFMBT1(0.0),RFT1(0.0),PRKCD(49.0) }\end{array}$ \\
\hline rs 13262595 & 8 & 143316970 & $1.585 \times 10^{-7}$ & 1.07573 & 0.0139 & $\mathrm{~A} / \mathrm{G}$ & 0.454 & 0.424 & 0.993 & LINC00051(0.0),TSNARE1(0.0) \\
\hline rs674094 & 11 & 57665336 & $1.844 \times 10^{-7}$ & 1.08622 & 0.0159 & $\mathrm{~A} / \mathrm{C}$ & 0.319 & 0.332 & 0.99 & $\begin{array}{l}\text { SERPING1(-233.0),MIR130A(-206.6), } \\
\text { YPEL4(-197.9),CLP1(-186.0), } \\
\text { ZDHHC5(-146.7),MED19(-135.5), } \\
\text { TMX2(-106.9),TMX2-CTNND1(-28.7), } \\
\text { SELENOH(-104.3),BTBD18(-96.1), } \\
\text { CTNND1(-28.3) }\end{array}$ \\
\hline rs79712033 & 2 & 147846501 & $1.999 \times 10^{-7}$ & 1.17975 & 0.0318 & $\mathrm{~A} / \mathrm{G}$ & 0.945 & 0.937 & 0.971 & - \\
\hline rs7219489 & 17 & 8121622 & $2.79 \times 10^{-7}$ & 1.08405 & 0.0157 & $\mathrm{G} / \mathrm{T}$ & 0.732 & 0.727 & 0.994 & $\begin{array}{l}\text { ALOXE3(-49.4),HES7(-44.2),PER1(-15.9), } \\
\text { MIR6883(-23.2),VAMP2(-5.4),TMEM107(0.0), } \\
\text { SNORD118(0.0),MIR4521(0.0),BORCS6(0.0), } \\
\text { AURKB(0.0),LINC00324(0.0),CTC1(0.0), } \\
\text { PFAS(0.0),SLC25A35(19.5),RANGRF(20.4), } \\
\text { ARHGEF15(41.9),ODF4(71.5) }\end{array}$ \\
\hline rs9535127 & 13 & 31606961 & $5.725 \times 10^{-7}$ & 1.07659 & 0.0148 & $\mathrm{~T} / \mathrm{C}$ & 0.487 & 0.49 & 0.993 & TEX26(-7.3) \\
\hline rs7128224 & 11 & 77360928 & $5.817 \times 10^{-7}$ & 1.0835 & 0.016 & $\mathrm{~T} / \mathrm{G}$ & 0.316 & 0.308 & 0.989 & $\begin{array}{l}\text { LOC646029(-24.2),AQP11(0.0),CLNS1A(0.0), } \\
\text { RSF1(0.0),AAMDC(121.2),INTS4(178.8), } \\
\text { KCTD14(315.8),NDUFC2-KCTD14(315.8) }\end{array}$ \\
\hline rs 424541 & 22 & 29863922 & $8.089 \times 10^{-7}$ & 0.91439 & 0.0181 & $\mathrm{C} / \mathrm{T}$ & 0.818 & 0.789 & 0.997 & $\begin{array}{l}\text { AP1B1(-29.4),RFPL1S(0.0), RFPL1(0.0), } \\
\text { NEFH(0.0),THOC5(0.0),NIPSNAP1(36.9), } \\
\text { NF2(85.6) }\end{array}$ \\
\hline rs34289388 & 2 & 208280861 & $9.928 \times 10^{-7}$ & 1.08741 & 0.0171 & G/A & 0.8 & 0.788 & 0.998 & - \\
\hline
\end{tabular}

Notes: SNP: single-nucleotide polymorphism; CHR: chromosome; BP: base pair position (hg19); P: p value; OR: odds ratio for allele1; A1/A2: effect allele/ reference allele; FRQ_A: frequency of allele1 in affected cases; FRQ_U: frequency of allele1 unaffected controls; INFO: imputation INFO score; genes.6.50kb(dist2index): list of genes within the region of LD-friends. 6 (variants with LD-r2 $>0.6$ to index SNP) $( \pm 50 \mathrm{~kb}$ ), in brackets distance to index SNP in kb. 
medRxiv preprint doi: https://doi.org/10.1101/2021.10.13.21261078; this version posted October 23, 2021. The copyright holder for this preprint (which was not certified by peer review) is the author/funder, who has granted medRxiv a license to display the preprint in perpetuity. It is made available under a CC-BY-NC-ND 4.0 International license .

Table 2. Genes significantly associated with OCD, as indicated by 4 gene-based methods

\begin{tabular}{|c|c|c|c|c|c|c|c|c|c|}
\hline \multirow[t]{2}{*}{ Locus } & \multirow[t]{2}{*}{ Top genes } & \multirow[t]{2}{*}{ BP start } & \multirow[t]{2}{*}{ BP stop } & \multirow[t]{2}{*}{ N SNPs } & \multirow[t]{2}{*}{ p value } & \multicolumn{4}{|c|}{ Gene-based method* } \\
\hline & & & & & & C-MAGMA & E-MAGMA & H-MAGMA & S-MultiXcan \\
\hline \multirow[t]{4}{*}{$3 \mathrm{p} 21.1$} & SPCS1 & 52738971 & 52745162 & 59 & $2.57 \times 10^{-7}$ & & & & \\
\hline & NEK4 & 52742465 & 52804965 & 43 & $1.47 \times 10^{-6}$ & & & & \\
\hline & TMEM110 & 52870772 & 52931612 & 34 & $1.04 \times 10^{-6}$ & & & & \\
\hline & SFMBT1 & 52933221 & 53080089 & 274 & $2.83 \times 10^{-7}$ & & & & \\
\hline $9 q 33.3$ & RPL35 & 127620158 & 127624260 & N/A & $1.01 \times 10^{-6}$ & & & & \\
\hline $9 \mathrm{q} 33.3$ & HSPA5 & 127997132 & 128003609 & 72 & $5.49 \times 10^{-7}$ & & & & \\
\hline $11 \mathrm{q} 12.1$ & TIMM10 & 57295936 & 57298276 & 27 & $3.73 \times 10^{-7}$ & & & & \\
\hline \multirow[t]{2}{*}{$12 q 24.23$} & LOC101928274 & 118501398 & 118541753 & 209 & $1.22 \times 10^{-6}$ & & & & \\
\hline & VSIG10 & 118501398 & 118541753 & 209 & $1.22 \times 10^{-6}$ & & & & \\
\hline $13 q 12.3$ & MEDAG & 31480328 & 31499709 & 84 & $1.07 \times 10^{-7}$ & & & & \\
\hline $13 q 13.3$ & POSTN & 38136720 & 38172981 & 333 & $1.52 \times 10^{-6}$ & & & & \\
\hline $17 \mathrm{p} 13.1$ & ACAP1 & 7239848 & 7254797 & 105 & $2.47 \times 10^{-8}$ & & & & \\
\hline \multirow[t]{4}{*}{$17 \mathrm{p} 13.1$} & PER1 & 8043788 & 8059723 & 30 & $8.89 \times 10^{-8}$ & & & & \\
\hline & TMEM107 & 8076555 & 8079717 & 13 & $1.39 \times 10^{-7}$ & & & & \\
\hline & SNORD118 & 8076772 & 8076905 & 7 & $2.38 \times 10^{-7}$ & & & & \\
\hline & BORCS6 & 8091651 & 8094225 & 14 & $6.63 \times 10^{-8}$ & & & & \\
\hline $17 \mathrm{p} 13.1$ & CTC1 & 8128139 & 8151413 & 134 & $2.30 \times 10^{-7}$ & & & & \\
\hline $22 \mathrm{q} 12.2$ & NEFH & 29876181 & 29887279 & 27 & $4.74 \times 10^{-7}$ & & & & \\
\hline
\end{tabular}

Notes: P values thresholds for each gene-based method was calculated using Bonferroni correction, for the total number of tests performed for a given method. The $\mathrm{P}$ value for the most significant gene-based association is reported in the Table. C-MAGMA: $2.50 \times 10^{-6}$ ( $\mathrm{N}=20,031$ tests); E-MAGMA: $\mathrm{P}<2.51 \times 10^{-7}$ ( $\mathrm{N}=199,421$ tests); H-MAGMA: $\mathrm{P}<2.05 \times 10^{-6}$ ( $\mathrm{N}=24,358$ tests); $\mathrm{S}-$ MultiXcan: $\mathrm{P}<2.31 \times 10^{-6}$ $(\mathrm{N}=21,601$ tests). The table describes the locus, top gene-based associations within each locus (top genes), location of the associated gene in base pairs (hg19) (BP start and BP stop, respectively), the number of SNPs included in the model (N SNPs), ae.g., .* Black shading indicates the method with the most significant gene-based $\mathrm{P}$ value; grey shading indicates a significant association for the same gene using a different gene-based method. 
medRxiv preprint doi: https://doi.org/10.1101/2021.10.13.21261078; this version posted October 23, 2021. The copyright holder for this preprint (which was not certified by peer review) is the author/funder, who has granted medRxiv a license to display the preprint in perpetuity.

It is made available under a CC-BY-NC-ND 4.0 International license .

Fig. 1. Manhattan plot of the results from the GWAS meta-analysis of OCD: The $y$-axis represents -log10 $p$ values for association of variants with OCD, from metaanalysis using an inverse-variance weighted fixed effects model. The x-axis represents chromosomes 1 to 22 . The horizontal red line represents the threshold for genome-wide significance. The index variant of the one genome-wide significant locus is highlighted as a green diamond.

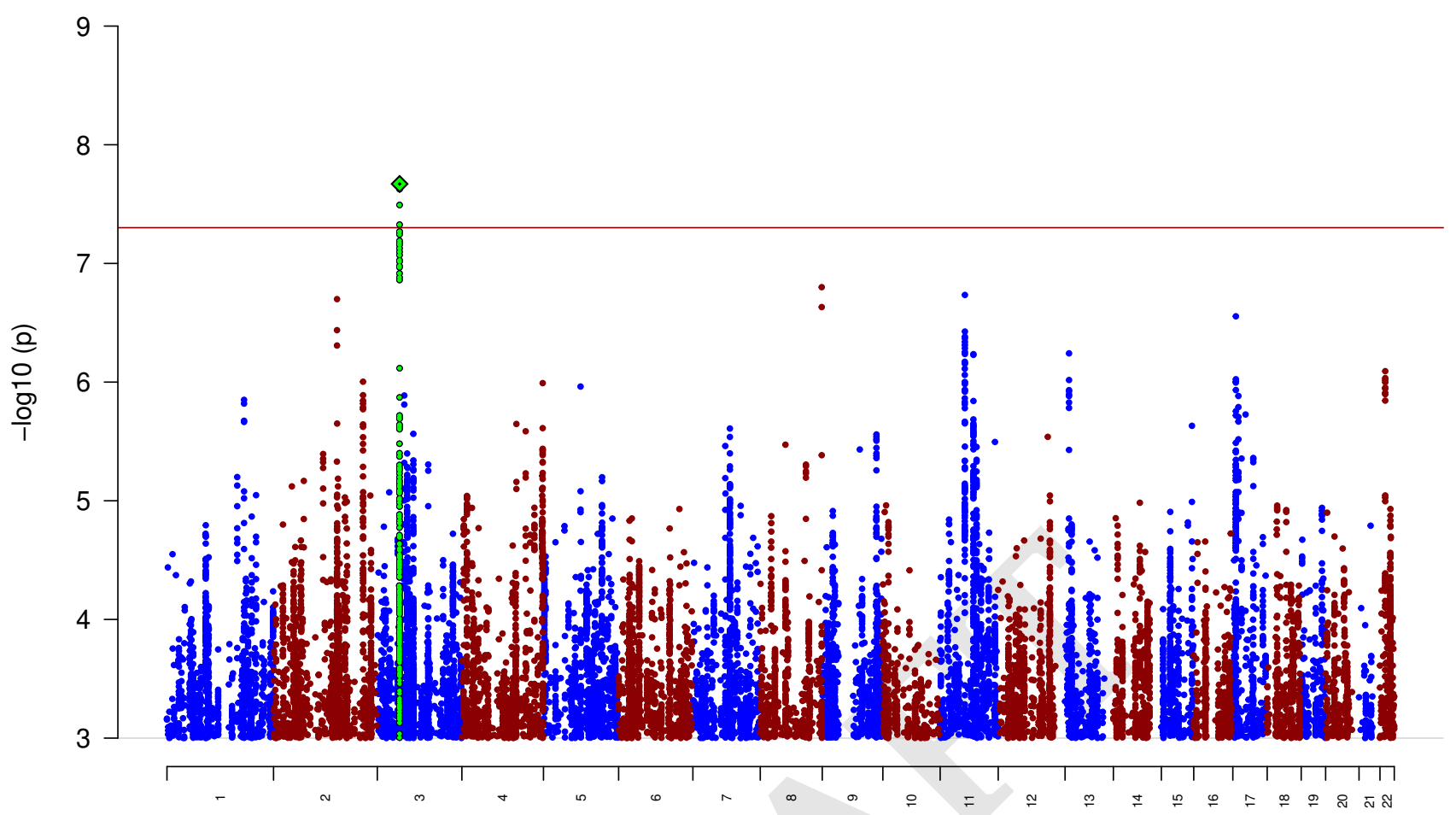

Chromosome 
medRxiv preprint doi: https://doi.org/10.1101/2021.10.13.21261078; this version posted October 23, 2021. The copyright holder for this preprint (which was not certified by peer review) is the author/funder, who has granted medRxiv a license to display the preprint in perpetuity.

It is made available under a CC-BY-NC-ND 4.0 International license .

Fig. 2. Regional plot of the genome-wide significant locus rs2581789. The - $\log _{10}$ ( $p$-value) of SNPs in the OCD meta-analysis GWAS is shown on the left $y$ axis. The recombination rates expressed in centimorgans (cM) per Mb (Megabase) (blue line) are shown on the right $y$ axis. Position in Mb is on the $x$ axis. Only the SNPs with association $p$-value less than 0.1 were plotted. The most associated SNP is shown as a purple diamond.

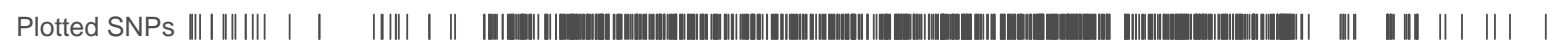

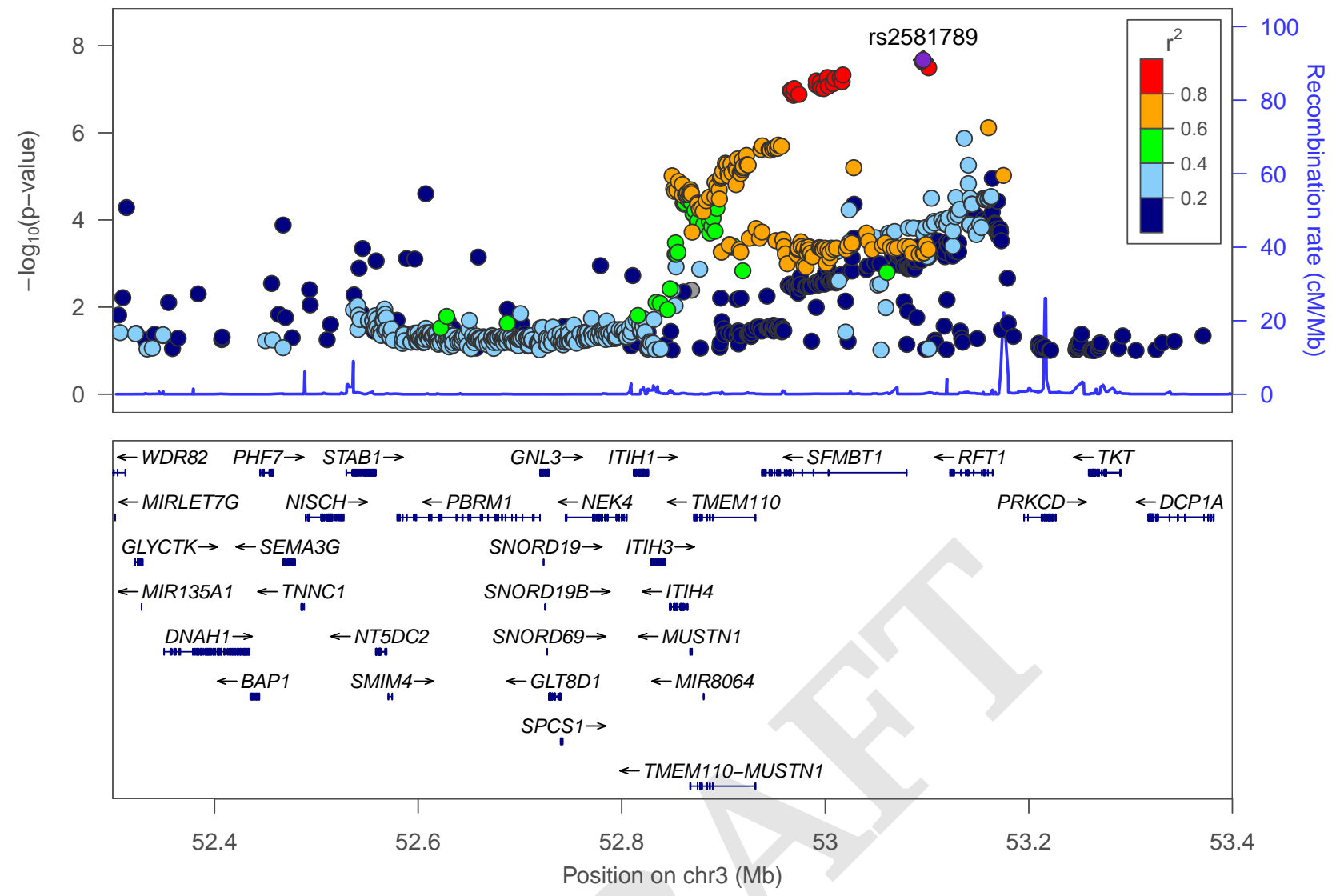


medRxiv preprint doi: https://doi.org/10.1101/2021.10.13.21261078; this version posted October 23, 2021. The copyright holder for this preprint (which was not certified by peer review) is the author/funder, who has granted medRxiv a license to display the preprint in perpetuity.

It is made available under a CC-BY-NC-ND 4.0 International license.

Fig. 3. Forest plot of SNP rs13262595. The plot shows the effect estimate with $95 \%$-confidence interval for each cohort contributing to the meta-analysis and for the inverse variance weighted meta analysis. The table lists INFO (imputation score), p-value, f_ca(n) (frequency cases), f_co(n) (frequency controls), In(OR), and STDerr (standard error) for each of the contributing cohorts and for the meta-analysis. At the top, + indicates a positive direction of effect, - a negative direction of effect while ? indicates that the SNP was not contained in the respective cohort.

rs2581789

IOCDF-GC TR

AUS

DOTS

EPOC

Estonia

FinnGen

HUNT

$\mathrm{MoBa}$

NORDiC-nor

NORDiC-swe_EGOS

UKBB

IOCDF-GC CC

OCGAS

Psych_Broad

WWF

Meta - analysis
C/T

p_value

1.01

0.99

0.98

0.99

1.00

1.00

0.99

1.00

1.01

0.99

0.99

0.98

1.01

0.99

1.01

1.00

0.426

0.227
3:53096230

$$
\text { het_P: }
$$

f_ca(n)

$$
0.181
$$

het_l:

24.7

f_co(n) In(OR) STDerr

$0.111 \quad 0.317(323)$

$0.9 \quad 0.338(757)$

$0.0826 \quad 0.332(2678)$

$0.343(195)$

$0.385(493)$

$0.313(790) \quad 0.335(161390)$

$0.353(284) \quad 0.325(66192)$

$0.341(104) \quad 0.311(2193)$

$0.301(365) \quad 0.355(315)$

$0.328(1997) \quad 0.340(3943)$

$0.307(776) \quad 0.337(125729)$

$0.310(1519) \quad 0.344(3541)$

$0.318(986) \quad 0.359(1023)$

$0.347(1396) \quad 0.382(4009)$

$0.289(140)$

$0.344(526)$

$2.14 \mathrm{e}-08 \quad 0.328(14140) \quad 0.353(562117) \quad-0.0869 \quad 0.0155$

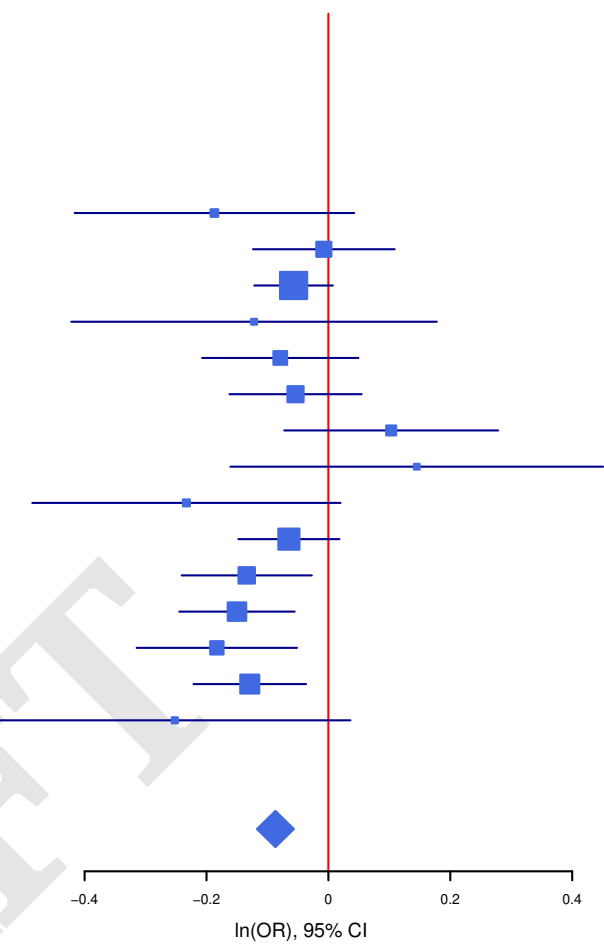


medRxiv preprint doi: https://doi.org/10.1101/2021.10.13.21261078; this version posted October 23, 2021. The copyright holder for this preprint (which was not certified by peer review) is the author/funder, who has granted medRxiv a license to display the preprint in perpetuity.

\section{It is made available under a CC-BY-NC-ND 4.0 International license .}

Fig. 4. Genetic correlations ( $\mathbf{r g})$ between $\mathrm{OCD}$ and a broad range $(\mathbf{N}=\mathbf{8 2})$ of other phenotypes. Error bars represent $95 \%$ confidence intervals and asterisks indicate significant associations after FDR correction for multiple testing. Non-significant correlations with SE $>0.5$ are excluded from display.
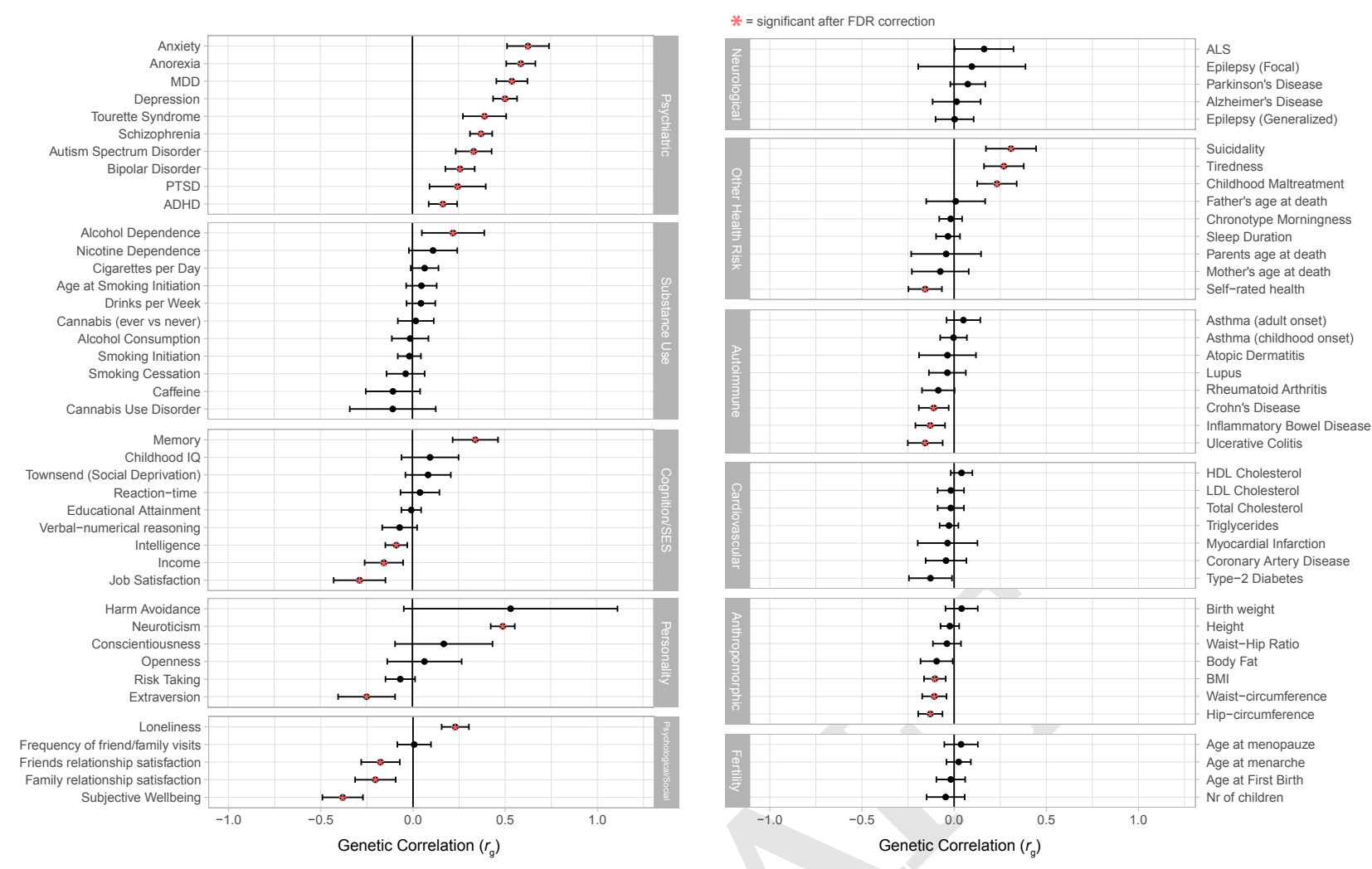